\title{
Stability of Levitating Objects
}

\author{
Boris V. Alexeev \\ Moscow Technological University, 119571 Moscow, prospect Vernadskogo 86, Russia \\ E-mail: Boris.Vlad.Alexeev@gmail.com
}

\begin{abstract}
In this paper we revisit the levitation phenomenon using the nonlocal physical description of this levitation phenomenon. This approach can identify the conditions when the levitation can take place under the influence of correlated electromagnetic and gravitational fields. The sufficient mathematical conditions of levitation are used. It means that the regime of levitation could be realized from the position of the non-local hydrodynamics. The stability of the spherical levitating objects is investigated. The appearance of the strong radial fluctuations leads to the destruction of the levitation regime.
\end{abstract}

Keywords: Foundations of the theory of transport processes, generalized hydrodynamic Alexeev equations, basements of non-local physics, levitation, Searl effect generator

\section{Introduction}

In this paper we revisit the levitation phenomenon using nonlocal kinetic theory (developed in particular in $[1-4])$ in the application to the levitation phenomenon.

The investigations of the levitation stability have a long history and are considered in details in [5-10]. As usual the problem review begins with the citation of the Earnshaw paper [5]. Earnshaw's theorem depends on a mathematical property of the $1 / r$ type energy potential valid for magnetostatic and electrostatic events and gravitation. At any point where there is force balance equal to zero, the equilibrium is unstable because there can be no local minimum in the potential energy. There must be some loopholes though, because magnets above superconductors and the magnet configuration do stably levitate including frogs [7] and toys like levitron (spinning magnet tops), flying globe and so on [11]. It means that diamagnetic material can stabilize the levitation of permanent magnets. It is well known that the potential energy density of the magnetic field can be written as

$$
w_{m}=-\mathbf{M} \cdot \mathbf{B}
$$

where $\mathbf{B}$ is magnetic induction, $\mathbf{M}$ is magnetization. Using the phenomenological relation

$$
\mathbf{M}=\chi \mathbf{H}
$$

where $\chi$ is magnetic susceptibility, we have for the unit volume of a magnetic material

$$
w_{m}=-\frac{\chi}{\mu \mu_{0}} B^{2}
$$

The force acting on the unit volume of a levitating object is

$$
\mathbf{F}=\frac{\chi}{\mu_{0} \mu} \operatorname{grad} B^{2}
$$

if the phenomenological parameters are constant. Diamagnets (for which $\chi<0$ ) are repelled by magnetic fields and attracted to field minima. As a result, diamagnets can satisfy the stability conditions [6 - 9] and the following conditions are exceptions to Earnshaw's theorem:

a) Diamagnetism occurs in materials which have a relative permeability less than one. The result is that eddy currents are induced in a diamagnetic material, it will repel magnetic flux.

b) The Meissner effect occurs in superconductors. Superconductors have zero internal resistance. As such induced currents tend to persist, and as a result the magnetic field they cause will persist as well.

c) As result of oscillations, when an alternating current is passed through an electromagnet, it behaves like a diamagnetic material.

d) Rotation: employed by the Levitron, it uses gyroscopic motion to overcome levitation instability. 
e) Feedback can be used in conjunction with electromagnets to dynamically adjust magnetic flux in order to maintain levitation.

The main shortcoming of the Earnshaw theory consists in application of principles of local physics to the non-equilibrium non-local statistical systems.

The aim of this paper consists in application of the non-local physics methods (obtained in the frame of the unified non-local theory of transport processes (UNT)) to the effect of levitation. We intend to answer two questions:

1) Is it possible using the sufficient conditions of levitation (see UNT, for example, in [12-15]) to obtain the stable levitating object?

2) Is it possible to speak about the destruction of the radial stability of the levitating spherical object as a result of the strong radial fluctuations?

\section{Basic Equations}

Non-local hydrodynamic equations have the form [1 - 4]:

(continuity equation for a mixture)

$$
\begin{aligned}
& \frac{\partial}{\partial t}\left\{\rho-\sum_{\alpha} \tau_{\alpha}^{(0)}\left[\frac{\partial \rho_{\alpha}}{\partial t}+\frac{\partial}{\partial \mathbf{r}} \cdot\left(\rho_{\alpha} \mathbf{v}_{0}\right)\right]\right\} \\
& +\frac{\partial}{\partial \mathbf{r}} \cdot\left\{\rho \mathbf{v}_{0}-\sum_{\alpha} \tau_{\alpha}^{(0)}\left[\frac{\partial}{\partial t}\left(\rho_{\alpha} \mathbf{v}_{0}\right)+\frac{\partial}{\partial \mathbf{r}} \cdot\left(\rho_{\alpha} \mathbf{v}_{0} \mathbf{v}_{0}\right)+\overrightarrow{\mathrm{I}} \cdot \frac{\partial p_{\alpha}}{\partial \mathbf{r}}-\rho_{\alpha} \mathbf{F}_{\alpha}^{(1)}-\frac{q_{\alpha}}{m_{\alpha}} \rho_{\alpha} \mathbf{v}_{0} \times \mathbf{B}\right]\right\}=0,
\end{aligned}
$$

(motion equation)

$$
\begin{aligned}
& \frac{\partial}{\partial t}\left\{\rho \mathbf{v}_{0}-\sum_{\alpha} \tau_{\alpha}^{(0)}\left[\frac{\partial}{\partial t}\left(\rho_{\alpha} \mathbf{v}_{0}\right)+\frac{\partial}{\partial \mathbf{r}} \cdot \rho_{\alpha} \mathbf{v}_{0} \mathbf{v}_{0}+\frac{\partial p_{\alpha}}{\partial \mathbf{r}}-\rho_{\alpha} \mathbf{F}_{\alpha}^{(1)}-\frac{q_{\alpha}}{m_{\alpha}} \rho_{\alpha} \mathbf{v}_{0} \times \mathbf{B}\right]\right\} \\
& -\sum_{\alpha} \mathbf{F}_{\alpha}^{(1)}\left[\rho_{\alpha}-\tau_{\alpha}^{(0)}\left(\frac{\partial \rho_{\alpha}}{\partial t}+\frac{\partial}{\partial \mathbf{r}} \cdot\left(\rho_{\alpha} \mathbf{v}_{0}\right)\right]\right]-\sum_{\alpha} \frac{q_{\alpha}}{m_{\alpha}}\left\{\rho_{\alpha} \mathbf{v}_{0}-\tau_{\alpha}^{(0)}\left[\frac{\partial}{\partial t}\left(\rho_{\alpha} \mathbf{v}_{0}\right)+\frac{\partial}{\partial \mathbf{r}} \cdot \rho_{\alpha} \mathbf{v}_{0} \mathbf{v}_{0}\right.\right. \\
& \left.\left.+\frac{\partial p_{\alpha}}{\partial \mathbf{r}}-\rho_{\alpha} \mathbf{F}_{\alpha}^{(1)}-\frac{q_{\alpha}}{m_{\alpha}} \rho_{\alpha} \mathbf{v}_{0} \times \mathbf{B}\right]\right\} \times \mathbf{B}+\frac{\partial}{\partial \mathbf{r}} \cdot\left\{\rho_{0} \mathbf{v}_{0}+p \overrightarrow{\mathrm{I}}-\sum_{\alpha} \tau_{\alpha}^{(0)}\left[\frac { \partial } { \partial t } \left(\rho_{\alpha} \mathbf{v}_{0} \mathbf{v}_{0}\right.\right.\right. \\
& \left.+p_{\alpha} \overrightarrow{\mathrm{I}}\right)+\frac{\partial}{\partial \mathbf{r}} \cdot \rho_{\alpha}\left(\mathbf{v}_{0} \mathbf{v}_{0}\right) \mathbf{v}_{0}+2 \overleftrightarrow{\mathrm{I}}\left(\frac{\partial}{\partial \mathbf{r}} \cdot\left(p_{\alpha} \mathbf{v}_{0}\right)\right)+\frac{\partial}{\partial \mathbf{r}} \cdot\left(\overleftrightarrow{\mathrm{I}} p_{\alpha} \mathbf{v}_{0}\right) \\
& \left.\left.-\mathbf{F}_{\alpha}^{(1)} \rho_{\alpha} \mathbf{v}_{0}-\rho_{\alpha} \mathbf{v}_{0} \mathbf{F}_{\alpha}^{(1)}-\frac{q_{\alpha}}{m_{\alpha}} \rho_{\alpha}\left[\mathbf{v}_{0} \times \mathbf{B}\right] \mathbf{v}_{0}-\frac{q_{\alpha}}{m_{\alpha}} \rho_{\alpha} \mathbf{v}_{0}\left[\mathbf{v}_{0} \times \mathbf{B}\right]\right]\right\}=0
\end{aligned}
$$

(energy equation) 


$$
\begin{aligned}
& \frac{\partial}{\partial t}\left\{\frac{\rho v_{0}^{2}}{2}+\frac{3}{2} p+\sum_{\alpha} \varepsilon_{\alpha} n_{\alpha}-\sum_{\alpha} \tau_{\alpha}^{(0)}\left[\frac{\partial}{\partial t}\left(\frac{\rho_{\alpha} v_{0}^{2}}{2}+\frac{3}{2} p_{\alpha}+\varepsilon_{\alpha} n_{\alpha}\right)\right.\right. \\
& \left.\left.+\frac{\partial}{\partial \mathbf{r}} \cdot\left(\frac{1}{2} \rho_{\alpha} v_{0}^{2} \mathbf{v}_{0}+\frac{5}{2} p_{\alpha} \mathbf{v}_{0}+\varepsilon_{\alpha} n_{\alpha} \mathbf{v}_{0}\right)-\mathbf{F}_{\alpha}^{(1)} \cdot \rho_{\alpha} \mathbf{v}_{0}\right]\right\} \\
& +\frac{\partial}{\partial \mathbf{r}} \cdot\left\{\frac{1}{2} \rho v_{0}^{2} \mathbf{v}_{0}+\frac{5}{2} p \mathbf{v}_{0}+\mathbf{v}_{0} \sum_{\alpha} \varepsilon_{\alpha} n_{\alpha}-\sum_{\alpha} \tau_{\alpha}^{(0)}\left[\frac{\partial}{\partial t}\left(\frac{1}{2} \rho_{\alpha} v_{0}^{2} \mathbf{v}_{0}+\frac{5}{2} p_{\alpha} \mathbf{v}_{0}+\varepsilon_{\alpha} n_{\alpha} \mathbf{v}_{0}\right)\right.\right. \\
& +\frac{\partial}{\partial \mathbf{r}} \cdot\left(\frac{1}{2} \rho_{\alpha} v_{0}^{2} \mathbf{v}_{0} \mathbf{v}_{0}+\frac{7}{2} p_{\alpha} \mathbf{v}_{0} \mathbf{v}_{0}+\frac{1}{2} p_{\alpha} v_{0}^{2} \overrightarrow{\mathrm{I}}+\frac{5}{2} \frac{p_{\alpha}^{2}}{\rho_{\alpha}} \overrightarrow{\mathrm{I}}+\varepsilon_{\alpha} n_{\alpha} \mathbf{v}_{0} \mathbf{v}_{0}+\varepsilon_{\alpha} \frac{p_{\alpha}}{m_{\alpha}}\right) \\
& -\rho_{\alpha} \mathbf{F}_{\alpha}^{(1)} \cdot \mathbf{v}_{0} \mathbf{v}_{0}-p_{\alpha} \mathbf{F}_{\alpha}^{(1)} \cdot \overrightarrow{\mathrm{I}}-\frac{1}{2} \rho_{\alpha} v_{0}^{2} \mathbf{F}_{\alpha}^{(1)}-\frac{3}{2} \mathbf{F}_{\alpha}^{(1)} p_{\alpha}-\frac{\rho_{\alpha} v_{0}^{2}}{2} \frac{q_{\alpha}}{m_{\alpha}}\left[\mathbf{v}_{0} \times \mathbf{B}\right] \\
& \left.\left.-\frac{5}{2} p_{\alpha} \frac{q_{\alpha}}{m_{\alpha}}\left[\mathbf{v}_{0} \times \mathbf{B}\right]-\varepsilon_{\alpha} n_{\alpha} \frac{q_{\alpha}}{m_{\alpha}}\left[\mathbf{v}_{0} \times \mathbf{B}\right]-\varepsilon_{\alpha} n_{\alpha} \mathbf{F}_{\alpha}^{(1)}\right]\right\}-\mathbf{v}_{0} \cdot \sum_{\alpha} \rho_{\alpha} \mathbf{F}_{\alpha}^{(1)} \\
& +\sum_{\alpha} \tau_{\alpha}^{(0)} \mathbf{F}_{\alpha}^{(1)} \cdot\left[\frac{\partial}{\partial t}\left(\rho_{\alpha} \mathbf{v}_{0}\right)+\frac{\partial}{\partial \mathbf{r}} \cdot \rho_{\alpha} \mathbf{v}_{0} \mathbf{v}_{0}+\frac{\partial}{\partial \mathbf{r}} \cdot p_{\alpha} \overrightarrow{\mathrm{I}}-\rho_{\alpha} \mathbf{F}_{\alpha}^{(1)}-q_{\alpha} n_{\alpha}\left[\mathbf{v}_{0} \times \mathbf{B}\right]\right]=0 .
\end{aligned}
$$

where $\mathbf{v}_{0}$ is the hydrodynamic velocity in the coordinate system at rest, $\rho_{\alpha}$ is the density of $\alpha$ - species, $p$ is the pressure, $\overrightarrow{\mathrm{I}}$ - unit tensore, $\mathbf{F}_{\alpha}^{(1)}$ is the force of the non-nonmagnetic origin acting on the unit of mass, $\varepsilon_{\alpha}$ is the internal energy of a particle of the $\alpha$-species, $\tau$ is non-local parameter.

Important remarks:

1. Equations (2.1) - (2.3) should be considered as local approximation of non-local equations (NLE) written in the hydrodynamic form. NLE include quantum hydrodynamics of Schrödinger - Madelung as a deep particular case [4] and can be applied in the frame of the unified theory from the atom scale to the Universe evolution.

2. The basic system contains the cross terms for the forces of the mass and electro-magneto-dynamic origin. It means that the fluctuation of the gravitational field leads to the electro- magneto dynamical fluctuations and verse versa.

Sufficient conditions of levitation can be obtained from Eqs. (2.1) - (2.3) after equalizing all terms containing forces to zero. Namely, from the continuity equation

$$
\frac{\partial}{\partial \mathbf{r}} \cdot\left\{\sum_{\alpha} \tau_{\alpha}^{(0)}\left[\rho_{\alpha} \mathbf{F}_{\alpha}^{(1)}+\frac{q_{\alpha}}{m_{\alpha}} \rho_{\alpha} \mathbf{v}_{0} \times \mathbf{B}\right]=0,\right.
$$

from the motion equation as follows

$$
\begin{aligned}
& \frac{\partial}{\partial t}\left\{\sum_{\alpha} \tau_{\alpha}^{(0)}\left[\rho_{\alpha} \mathbf{F}_{\alpha}^{(1)}+\frac{q_{\alpha}}{m_{\alpha}} \rho_{\alpha} \mathbf{v}_{0} \times \mathbf{B}\right]\right\}-\sum_{\alpha} \mathbf{F}_{\alpha}^{(1)}\left[\rho_{\alpha}-\tau_{\alpha}^{(0)}\left(\frac{\partial \rho_{\alpha}}{\partial t}+\frac{\partial}{\partial \mathbf{r}} \cdot\left(\rho_{\alpha} \mathbf{v}_{0}\right)\right]\right] \\
& -\sum_{\alpha} \frac{q_{\alpha}}{m_{\alpha}}\left\{\tau_{\alpha}^{(0)}\left[\rho_{\alpha} \mathbf{F}_{\alpha}^{(1)}+\frac{q_{\alpha}}{m_{\alpha}} \rho_{\alpha} \mathbf{v}_{0} \times \mathbf{B}\right]\right\} \times \mathbf{B} \\
& +\frac{\partial}{\partial \mathbf{r}} \cdot\left\{\sum_{\alpha} \tau_{\alpha}^{(0)}\left[\mathbf{F}_{\alpha}^{(1)} \rho_{\alpha} \mathbf{v}_{0}+\rho_{\alpha} \mathbf{v}_{0} \mathbf{F}_{\alpha}^{(1)}+\frac{q_{\alpha}}{m_{\alpha}} \rho_{\alpha}\left[\mathbf{v}_{0} \times \mathbf{B}\right] \mathbf{v}_{0}+\frac{q_{\alpha}}{m_{\alpha}} \rho_{\alpha} \mathbf{v}_{0}\left[\mathbf{v}_{0} \times \mathbf{B}\right]\right]\right\}=0,
\end{aligned}
$$

and from the energy equation we find 


$$
\begin{aligned}
& \frac{\partial}{\partial t} \sum_{\alpha} \tau_{\alpha}^{(0)}\left(\rho_{\alpha} \mathbf{F}_{\alpha}^{(1)} \cdot \mathbf{v}_{0}\right) \\
& +\frac{\partial}{\partial \mathbf{r}} \cdot\left\{\sum _ { \alpha } \tau _ { \alpha } ^ { ( 0 ) } \left[\rho_{\alpha} \mathbf{F}_{\alpha}^{(1)} \cdot \mathbf{v}_{0} \mathbf{v}_{0}+p_{\alpha} \mathbf{F}_{\alpha}^{(1)} \cdot \overrightarrow{\mathrm{I}}+\frac{1}{2} \rho_{\alpha} v_{0}^{2} \mathbf{F}_{\alpha}^{(1)}+\frac{3}{2} \mathbf{F}_{\alpha}^{(1)} p_{\alpha}+\frac{\rho_{\alpha} v_{0}^{2}}{2} \frac{q_{\alpha}}{m_{\alpha}}\left[\mathbf{v}_{0} \times \mathbf{B}\right]\right.\right. \\
& \left.\left.+\frac{5}{2} p_{\alpha} \frac{q_{\alpha}}{m_{\alpha}}\left[\mathbf{v}_{0} \times \mathbf{B}\right]+\varepsilon_{\alpha} n_{\alpha} \frac{q_{\alpha}}{m_{\alpha}}\left[\mathbf{v}_{0} \times \mathbf{B}\right]+\varepsilon_{\alpha} n_{\alpha} \mathbf{F}_{\alpha}^{(1)}\right]\right\} \\
& -\mathbf{v}_{0} \cdot \sum_{\alpha} \rho_{\alpha} \mathbf{F}_{\alpha}^{(1)}+\sum_{\alpha} \tau_{\alpha}^{(0)} \mathbf{F}_{\alpha}^{(1)} \cdot\left[\frac{\partial}{\partial t}\left(\rho_{\alpha} \mathbf{v}_{0}\right)+\frac{\partial}{\partial \mathbf{r}} \cdot \rho_{\alpha} \mathbf{v}_{0} \mathbf{v}_{0}+\frac{\partial}{\partial \mathbf{r}} \cdot p_{\alpha} \overrightarrow{\mathrm{I}}-\rho_{\alpha} \mathbf{F}_{\alpha}^{(1)}-q_{\alpha} n_{\alpha}\left[\mathbf{v}_{0} \times \mathbf{B}\right]\right]=0 .
\end{aligned}
$$

From Eq. (2.4) we have

$$
\sum_{\alpha} \tau_{\alpha}^{(0)}\left[\rho_{\alpha} \mathbf{F}_{\alpha}^{(1)}+\frac{q_{\alpha}}{m_{\alpha}} \rho_{\alpha} \mathbf{v}_{0} \times \mathbf{B}\right]=\mathbf{L}(t)
$$

or

$$
\sum_{\alpha} \tau_{\alpha}^{(0)}\left[\rho_{\alpha} \mathbf{F}_{\alpha}^{(1)}+\frac{q_{\alpha}}{m_{\alpha}} \rho_{\alpha} \mathbf{v}_{0} \times \mathbf{B}\right]=\mathbf{L}
$$

where $\mathbf{L}$ is constant vector. Let us introduce vector $\mathbf{L}_{\alpha}(t)$

$$
\mathbf{L}_{\alpha}(t)=\tau_{\alpha}^{(0)}\left[\rho_{\alpha} \mathbf{F}_{\alpha}^{(1)}+\frac{q_{\alpha}}{m_{\alpha}} \rho_{\alpha} \mathbf{v}_{0} \times \mathbf{B}\right],
$$

and rewrite now Eq. (2.5), which contains the density fluctuation [1]

$$
\rho_{\alpha}^{f l}=\tau_{\alpha}^{(0)}\left(\frac{\partial \rho_{\alpha}}{\partial t}+\frac{\partial}{\partial \mathbf{r}} \cdot\left(\rho_{\alpha} \mathbf{v}_{0}\right)\right)
$$

We have

$$
\begin{aligned}
& \frac{\partial}{\partial t} \mathbf{L}(t)-\sum_{\alpha} \mathbf{F}_{\alpha}^{(1)} \rho_{\alpha}^{a}-\sum_{\alpha} \frac{q_{\alpha}}{m_{\alpha}} \mathbf{L}_{\alpha}(t) \times \mathbf{B} \\
& +\mathbf{v}_{0}\left\{\frac{\partial}{\partial \mathbf{r}} \cdot \sum_{\alpha} \tau_{\alpha}^{(0)}\left[\mathbf{F}_{\alpha}^{(1)} \rho_{\alpha}+\frac{q_{\alpha}}{m_{\alpha}} \rho_{\alpha}\left[\mathbf{v}_{0} \times \mathbf{B}\right]\right]\right\}+\left(\sum_{\alpha} \tau_{\alpha}^{(0)}\left[\mathbf{F}_{\alpha}^{(1)} \rho_{\alpha}+\frac{q_{\alpha}}{m_{\alpha}} \rho_{\alpha}\left[\mathbf{v}_{0} \times \mathbf{B}\right]\right] \cdot \frac{\partial}{\partial \mathbf{r}}\right) \mathbf{v}_{0} \\
& +\sum_{\alpha} \tau_{\alpha}^{(0)}\left[\rho_{\alpha} \mathbf{F}_{\alpha}^{(1)}+\frac{q_{\alpha}}{m_{\alpha}} \rho_{\alpha}\left[\mathbf{v}_{0} \times \mathbf{B}\right]\right] \frac{\partial}{\partial \mathbf{r}} \cdot \mathbf{v}_{0}+\left(\mathbf{v}_{0} \cdot \frac{\partial}{\partial \mathbf{r}}\right)\left\{\sum_{\alpha} \tau_{\alpha}^{(0)}\left[\rho_{\alpha} \mathbf{F}_{\alpha}^{(1)}+\frac{q_{\alpha}}{m_{\alpha}} \rho_{\alpha}\left[\mathbf{v}_{0} \times \mathbf{B}\right]\right]\right\}=0,
\end{aligned}
$$

where

Using also (2.9), we find

$$
\rho_{\alpha}^{a}=\rho_{\alpha}-\rho_{\alpha}^{f l}
$$

$$
\sum_{\alpha} \mathbf{F}_{\alpha}^{(1)} \rho_{\alpha}^{a}=\frac{\partial}{\partial t} \mathbf{L}(t)-\sum_{\alpha} \frac{q_{\alpha}}{m_{\alpha}} \mathbf{L}_{\alpha}(t) \times \mathbf{B}+\left(\mathbf{L}(t) \cdot \frac{\partial}{\partial \mathbf{r}}\right) \mathbf{v}_{0}+\mathbf{L}(t)\left(\frac{\partial}{\partial \mathbf{r}} \cdot \mathbf{v}_{0}\right)+\left(\mathbf{v}_{0} \cdot \frac{\partial}{\partial \mathbf{r}}\right) \mathbf{L}(t)
$$

The vector product in Eq. (2.13) can be transformed as

$$
\mathbf{L}_{\alpha}(t) \times \mathbf{B}=\tau_{\alpha}^{(0)} \rho_{\alpha} \mathbf{F}_{\alpha}^{(1)} \times \mathbf{B}-\tau_{\alpha}^{(0)} q_{\alpha} n_{\alpha}\left[\mathbf{v}_{0} B^{2}-\mathbf{B}\left(\mathbf{v}_{0} \cdot \mathbf{B}\right)\right]
$$

where $q_{\alpha} n_{\alpha}$ is the charge of $\alpha$-species in the unit volume.

Taking into account the relations (2.10), (2.11), (2.12), we can realize the analogical transformation of the energy condition (2.6): 


$$
\begin{aligned}
& \mathbf{v}_{0} \cdot \sum_{\alpha} \mathbf{F}_{\alpha}^{(1)} \rho_{\alpha}^{a}=\frac{\partial}{\partial t} \sum_{\alpha} \tau_{\alpha}^{(0)}\left(\rho_{\alpha} \mathbf{F}_{\alpha}^{(1)} \cdot \mathbf{v}_{0}\right)+\sum_{\alpha} \tau_{\alpha}^{(0)} \mathbf{F}_{\alpha}^{(1)} \cdot\left[\rho_{\alpha} \frac{\partial \mathbf{v}_{0}}{\partial t}+\rho_{\alpha}\left(\mathbf{v}_{0} \cdot \frac{\partial}{\partial \mathbf{r}}\right) \mathbf{v}_{0}+\frac{\partial}{\partial \mathbf{r}} \cdot p_{\alpha} \overrightarrow{\mathrm{I}}\right] \\
& +\frac{\partial}{\partial \mathbf{r}} \cdot\left\{\sum_{\alpha} \tau_{\alpha}^{(0)}\left[\begin{array}{l}
\left.\rho_{\alpha} \mathbf{F}_{\alpha}^{(1)} \cdot \mathbf{v}_{0} \mathbf{v}_{0}+p_{\alpha} \mathbf{F}_{\alpha}^{(1)} \cdot \overrightarrow{\mathrm{I}}+\frac{1}{2} \rho_{\alpha} v_{0}^{2} \mathbf{F}_{\alpha}^{(1)}+\frac{3}{2} \mathbf{F}_{\alpha}^{(1)} p_{\alpha}+\frac{\rho_{\alpha} v_{0}^{2}}{2} \frac{q_{\alpha}}{m_{\alpha}}\left[\mathbf{v}_{0} \times \mathbf{B}\right]+\right] \\
+\frac{5}{2} p_{\alpha} \frac{q_{\alpha}}{m_{\alpha}}\left[\mathbf{v}_{0} \times \mathbf{B}\right]
\end{array}\right]\right\} \\
& +\frac{\partial}{\partial \mathbf{r}} \cdot \sum_{\alpha} \varepsilon_{\alpha} n_{\alpha} \mathbf{L}_{\alpha}-\sum_{\alpha} \tau_{\alpha}^{(0)} \mathbf{F}_{\alpha}^{(1)} \cdot \mathbf{L}_{\alpha}
\end{aligned}
$$

Equations (2.7), (2.13) and (2.15) define the system of the sufficient conditions for levitation.

The choice of the non-local parameter needs in the special consideration [1 - 4]. The system of equations (2.1)-(2.3) turns into the system of quantum hydrodynamic equations by the suitable choice of the non-local parameter $\tau$. The relation between $\tau$ and kinetic energy $[3,4]$ is used in quantum hydrodynamics

$$
\tau=H / m u^{2}
$$

where $u$ is the particle velocity, $H$ is the coefficient of proportionality which reflects the state of the physical system. In the simplest case $H$ is equal to the Plank constant $\hbar$ and the corresponding relation (2.16) correlates with the Heisenberg inequality. From the first glance the approximation (2.16) is distinguished radically from the kinetic relation known from the theory of the rarefied gases

$$
\tau=\prod^{v \rho} / p
$$

which is used for the calculation of the non-local parameter in the macroscopic hydrodynamic case $(v$ is the kinematic viscosity). But it is not a case. In quantum approximation the value $v^{q u}=\hbar / m$ has the dimension $\left[\mathrm{cm}^{2} / \mathrm{s}\right]$ and can be called as quantum viscosity, for the electron species $v^{q u}=\hbar / m_{e}=1.1577 \mathrm{~cm}^{2} / \mathrm{s}$. If we take into account the value $p / \rho \sim \overline{V^{2}}$, then the interrelation of (2.16) and (2.17) becomes obvious.

\section{Spherical Levitating Object}

Let us construct the theory of levitating objects in the frame of UNT. This theory leads to the two systems of the hydrodynamic equations:

1. The first one does not contain the forces at all. This situation can be realized if the second item takes place.

2. All sufficient conditions (2.7), (2.13) and (2.15) which define the physical system levitation are fulfilled. This second system uses the hydrodynamic values $\rho_{\alpha}, \mathbf{v}_{0}, p_{\alpha}$ (obtained from the first system) for defining the electro-magnetic field configuration.

This splitting of description leads to the significant simplification of the numerical calculations. Let us write down the nonlocal hydrodynamic equations in the spherical coordinate system corresponding to the Item 1.

As an example we write Eq. (2.1) for the case 


$$
\begin{aligned}
& \frac{\partial}{\partial t}\left\{\rho-\tau\left[\frac{\partial \rho}{\partial t}+\frac{1}{r^{2}} \frac{\partial\left(r^{2} \rho v_{0 r}\right)}{\partial r}+\frac{1}{r \sin \theta} \frac{\partial\left(\rho v_{0 \phi}\right)}{\partial \phi}+\frac{1}{r \sin \theta} \frac{\partial\left(\rho v_{0 \theta} \sin \theta\right)}{\partial \theta}\right]\right\} \\
& +\frac{1}{r^{2}} \frac{\partial}{\partial r}\left\{r ^ { 2 } \left\{\rho v_{0 r}-\tau\left[\frac{\partial}{\partial t}\left(\rho v_{0 r}\right)+\frac{1}{r^{2}} \frac{\partial\left(r^{2} \rho v_{0 r}^{2}\right)}{\partial r}+\frac{1}{r \sin \theta} \frac{\partial\left(\rho v_{0 \phi} v_{0 r}\right)}{\partial \phi}\right.\right.\right. \\
& \left.\left.\left.+\frac{1}{r \sin \theta} \frac{\partial\left(\rho v_{0 \theta} v_{0 r} \sin \theta\right)}{\partial \theta}-\rho g_{r}\right]\right\}\right\}+\frac{1}{r \sin \theta} \frac{\partial}{\partial \phi}\left\{\rho v_{0 \phi}-\tau\left[\frac{\partial}{\partial t}\left(\rho v_{0 \phi}\right)+\frac{1}{r^{2}} \frac{\partial\left(r^{2} \rho v_{0 r} v_{0 \phi}\right)}{\partial r}\right.\right. \\
& \left.\left.+\frac{1}{r \sin \theta} \frac{\partial\left(\rho v_{0 \phi}^{2}\right)}{\partial \phi}+\frac{1}{r \sin \theta} \frac{\partial\left(\rho v_{0 \theta} v_{0 \phi} \sin \theta\right)}{\partial \theta}-\rho g_{\phi}\right]\right\}+\frac{1}{r \sin \theta} \frac{\partial}{\partial \theta}\left\{\operatorname { s i n } \theta \left\{\rho v_{0 \theta}-\tau\left[\frac{\partial}{\partial t}\left(\rho v_{0 \theta}\right)\right.\right.\right. \\
& \left.\left.\left.+\frac{1}{r^{2}} \frac{\partial\left(r^{2} \rho v_{0 r} v_{0 \theta}\right)}{\partial r}+\frac{1}{r \sin \theta} \frac{\partial\left(\rho v_{0 \phi} v_{0 \theta}\right)}{\partial \phi}+\frac{1}{r \sin \theta} \frac{\partial\left(\rho v_{0 \theta}^{2} \sin \theta\right)}{\partial \theta}-\rho g_{\theta}\right]\right\}\right\} \\
& -\frac{1}{r^{2}} \frac{\partial}{\partial r}\left(\tau r^{2} \frac{\partial p}{\partial r}\right)-\frac{1}{r^{2} \sin \theta} \frac{\partial}{\partial \theta}\left(\tau \sin \theta \frac{\partial p}{\partial \theta}\right)-\frac{1}{r^{2} \sin ^{2} \theta} \frac{\partial}{\partial \phi}\left(\tau \frac{\partial p}{\partial \phi}\right)=0
\end{aligned}
$$

The full system of equations for the multi-component species (containing also particles with the internal energies) in the spherical coordinate system can be found in $[3,4]$. Here we write the simplified system of equations for the one component physical system with the azimuthally $\phi$ - symmetry.

Continuity equation

$$
\begin{aligned}
& \frac{\partial}{\partial t}\left\{\rho-\tau\left[\frac{\partial \rho}{\partial t}+\frac{1}{r^{2}} \frac{\partial\left(r^{2} \rho v_{0 r}\right)}{\partial r}+\frac{1}{r \sin \theta} \frac{\partial\left(\rho v_{0 \theta} \sin \theta\right)}{\partial \theta}\right]\right\} \\
& +\frac{1}{r^{2}} \frac{\partial}{\partial r}\left\{r^{2}\left\{\rho v_{0 r}-\tau\left[\frac{\partial}{\partial t}\left(\rho v_{0 r}\right)+\frac{1}{r^{2}} \frac{\partial\left(r^{2} \rho v_{0 r}^{2}\right)}{\partial r}+\frac{1}{r \sin \theta} \frac{\partial\left(\rho v_{0 \theta} v_{0 r} \sin \theta\right)}{\partial \theta}\right]\right\}\right\} \\
& +\frac{1}{r \sin \theta} \frac{\partial}{\partial \theta}\left\{\sin \theta\left\{\rho v_{0 \theta}-\tau\left[\frac{\partial}{\partial t}\left(\rho v_{0 \theta}\right)+\frac{1}{r^{2}} \frac{\partial\left(r^{2} \rho v_{0 r} v_{0 \theta}\right)}{\partial r}+\frac{1}{r \sin \theta} \frac{\partial\left(\rho v_{0 \theta}^{2} \sin \theta\right)}{\partial \theta}\right]\right\}\right\} \\
& -\frac{1}{r^{2}} \frac{\partial}{\partial r}\left(\tau r^{2} \frac{\partial p}{\partial r}\right)-\frac{1}{r^{2} \sin \theta} \frac{\partial}{\partial \theta}\left(\tau \sin \theta \frac{\partial p}{\partial \theta}\right)=0
\end{aligned}
$$

Momentum equation (radial $r$ - component) 


$$
\begin{aligned}
& \frac{\partial}{\partial t}\left\{\rho v_{0 r}-\tau\left[\frac{\partial}{\partial t}\left(\rho v_{0 r}\right)+\frac{1}{r^{2}} \frac{\partial\left(r^{2} \rho v_{0 r}^{2}\right)}{\partial r}+\frac{1}{r \sin \theta} \frac{\partial\left(\rho v_{0 \theta} v_{0 r} \sin \theta\right)}{\partial \theta}+\frac{\partial p}{\partial r}\right]\right\} \\
& +\frac{1}{r^{2}} \frac{\partial}{\partial r}\left\{r^{2}\left\{\rho v_{0 r}^{2}-\tau\left[\frac{\partial}{\partial t}\left(\rho v_{0 r}^{2}\right)+\frac{1}{r^{2}} \frac{\partial\left(r^{2} \rho v_{0 r}^{3}\right)}{\partial r}+\frac{1}{r \sin \theta} \frac{\partial\left(\rho v_{0 \theta} v_{0 r}^{2} \sin \theta\right)}{\partial \theta}\right]\right\}\right\} \\
& +\frac{1}{r \sin \theta} \frac{\partial}{\partial \theta}\left\{\sin \theta\left\{\rho v_{0 \theta} v_{0 r}-\tau\left[\frac{\partial}{\partial t}\left(\rho v_{0 \theta} v_{0 r}\right)+\frac{1}{r^{2}} \frac{\partial\left(r^{2} \rho v_{0 \theta} v_{0 r}^{2}\right)}{\partial r}++\frac{1}{r \sin \theta} \frac{\partial\left(\rho v_{0 \theta}^{2} v_{0 r} \sin \theta\right)}{\partial \theta}\right]\right\}\right\} \\
& +\frac{\partial p}{\partial r}-\frac{\partial}{\partial r}\left(\tau \frac{\partial p}{\partial t}\right)-2 \frac{\partial}{\partial r}\left(\tau\left(\frac{1}{r^{2}} \frac{\partial\left(r^{2} p v_{0 r}\right)}{\partial r}+\frac{1}{r \sin \theta} \frac{\partial\left(p v_{0 \theta} \sin \theta\right)}{\partial \theta}\right)\right) \\
& -\frac{1}{r^{2}} \frac{\partial}{\partial r}\left(\tau r^{2} \frac{\partial\left(p v_{0 r}\right)}{\partial r}\right)-\frac{1}{r^{2} \sin \theta} \frac{\partial}{\partial \theta}\left(\tau \sin \theta \frac{\partial\left(p v_{0 r}\right)}{\partial \theta}\right)=0
\end{aligned}
$$

Momentum equation (angle $\theta$ - component)

$$
\begin{aligned}
& \frac{\partial}{\partial t}\left\{\rho v_{0 \theta}-\tau\left[\frac{\partial}{\partial t}\left(\rho v_{0 \theta}\right)+\frac{1}{r^{2}} \frac{\partial\left(r^{2} \rho v_{0 r} v_{0 \theta}\right)}{\partial r}+\frac{1}{r \sin \theta} \frac{\partial\left(\rho v_{0 \theta}^{2} \sin \theta\right)}{\partial \theta}+\frac{1}{r} \frac{\partial p}{\partial \theta}\right]\right\} \\
& +\frac{1}{r^{2}} \frac{\partial}{\partial r}\left\{r^{2}\left\{\rho v_{0 r} v_{0 \theta}-\tau\left[\frac{\partial}{\partial t}\left(\rho v_{0 r} v_{0 \theta}\right)+\frac{1}{r^{2}} \frac{\partial\left(r^{2} \rho v_{0 r}^{2} v_{0 \theta}\right)}{\partial r}+\frac{1}{r \sin \theta} \frac{\partial\left(\rho v_{0 \theta}^{2} v_{o r} \sin \theta\right)}{\partial \theta}\right]\right\}\right\} \\
& +\frac{1}{r \sin \theta} \frac{\partial}{\partial \theta}\left\{\operatorname { s i n } \theta \left\{\rho v_{0 \theta}^{2}-\tau\left[\frac{\partial}{\partial t}\left(\rho v_{0 \theta}^{2}\right)+\frac{1}{r^{2}} \frac{\partial\left(r^{2} \rho v_{0 r} v_{0 \theta}^{2}\right)}{\partial r}\right.\right.\right. \\
& \left.\left.\left.+\frac{1}{r \sin \theta} \frac{\partial\left(\rho v_{0 \theta}^{3} \sin \theta\right)}{\partial \theta}\right]\right\}\right\}+\frac{1}{r} \frac{\partial p}{\partial \theta}-\frac{1}{r} \frac{\partial}{\partial \theta}\left(\tau \frac{\partial p}{\partial t}\right)-\frac{2}{r} \frac{\partial}{\partial \theta}\left(\tau\left(\frac{1}{r^{2}} \frac{\partial\left(r^{2} p v_{0 r}\right)}{\partial r}+\frac{1}{r \sin \theta} \frac{\partial\left(p v_{0 \theta} \sin \theta\right)}{\partial \theta}\right)\right) \\
& -\frac{1}{r^{2}} \frac{\partial}{\partial r}\left(\tau r^{2} \frac{\partial\left(p v_{0 \theta}\right)}{\partial r}\right)-\frac{1}{r^{2} \sin \theta} \frac{\partial}{\partial \theta}\left(\tau \sin \theta \frac{\partial\left(p v_{0 \theta}\right)}{\partial \theta}\right)=0
\end{aligned}
$$

Energy equation

$$
\begin{aligned}
& \frac{\partial}{\partial t}\left\{\frac{1}{2} \rho v_{0}^{2}+\frac{3}{2} p-\tau\left[\frac{\partial}{\partial t}\left(\frac{1}{2} \rho v_{0}^{2}+\frac{3}{2} p\right)+\frac{1}{r^{2}} \frac{\partial}{\partial r}\left(r^{2} v_{0 r}\left(\frac{1}{2} \rho v_{0}^{2}+\frac{5}{2} p\right)\right)+\frac{1}{r \sin \theta} \frac{\partial}{\partial \theta}\left(\sin \theta v_{0 \theta}\left(\frac{1}{2} \rho v_{0}^{2}+\frac{5}{2} p\right)\right)\right]\right\} \\
& +\frac{1}{r^{2}} \frac{\partial}{\partial r}\left\{r ^ { 2 } \left\{\left(\frac{1}{2} \rho v_{0}^{2}+\frac{5}{2} p\right) v_{0 r}-\tau\left[\frac{\partial}{\partial t}\left(\left(\frac{1}{2} \rho v_{0}^{2}+\frac{5}{2} p\right) v_{0 r}\right)+\frac{1}{r^{2}} \frac{\partial}{\partial r}\left(r^{2}\left(\frac{1}{2} \rho v_{0}^{2}+\frac{7}{2} p\right) v_{0 r}^{2}\right)\right.\right.\right. \\
& \left.\left.\left.+\frac{1}{r \sin \theta} \frac{\partial}{\partial \theta}\left(\sin \theta\left(\frac{1}{2} \rho v_{0}^{2}+\frac{7}{2} p\right) v_{0 \theta} v_{0 r}\right)\right]\right\}\right\}+\frac{1}{r \sin \theta} \frac{\partial}{\partial \theta}\left\{\operatorname { s i n } \theta \left\{\left(\frac{1}{2} \rho v_{0}^{2}+\frac{5}{2} p\right) v_{0 \theta}\right.\right. \\
& \left.\left.-\tau\left[\frac{\partial}{\partial t}\left(\left(\frac{1}{2} \rho v_{0}^{2}+\frac{5}{2} p\right) v_{0 \theta}\right)+\frac{1}{r^{2}} \frac{\partial}{\partial r}\left(r^{2}\left(\frac{1}{2} \rho v_{0}^{2}+\frac{7}{2} p\right) v_{0 r} v_{0 \theta}\right)+\frac{1}{r \sin \theta} \frac{\partial}{\partial \theta}\left(\sin \theta\left(\frac{1}{2} \rho v_{0}^{2}+\frac{7}{2} p\right) v_{0 \theta}^{2}\right)\right]\right\}\right\} \\
& -\frac{1}{r^{2} \sin \theta} \frac{\partial}{\partial \theta}\left(\tau \sin \theta \frac{\partial}{\partial \theta}\left(\frac{1}{2} p v_{0}^{2}+\frac{5}{2} \frac{p^{2}}{\rho}\right)\right)=0
\end{aligned}
$$

For the full spherical symmetry, when only $r$ - dependence takes place we have:

Continuity equation 


$$
\begin{aligned}
& \frac{\partial}{\partial t}\left\{\rho-\tau\left[\frac{\partial \rho}{\partial t}+\frac{1}{r^{2}} \frac{\partial\left(r^{2} \rho v_{0 r}\right)}{\partial r}\right]\right\} \\
& +\frac{1}{r^{2}} \frac{\partial}{\partial r}\left\{r^{2}\left\{\rho v_{0 r}-\tau\left[\frac{\partial}{\partial t}\left(\rho v_{0 r}\right)+\frac{1}{r^{2}} \frac{\partial\left(r^{2} \rho v_{0 r}^{2}\right)}{\partial r}\right]\right\}\right\}-\frac{1}{r^{2}} \frac{\partial}{\partial r}\left(\tau r^{2} \frac{\partial p}{\partial r}\right)=0
\end{aligned}
$$

Momentum equation

$$
\begin{aligned}
& \frac{\partial}{\partial t}\left\{\rho v_{0 r}-\tau\left[\frac{\partial}{\partial t}\left(\rho v_{0 r}\right)+\frac{1}{r^{2}} \frac{\partial\left(r^{2} \rho v_{0 r}^{2}\right)}{\partial r}+\frac{\partial p}{\partial r}\right]\right\}+\frac{1}{r^{2}} \frac{\partial}{\partial r}\left\{r^{2}\left\{\rho v_{0 r}^{2}-\tau\left[\frac{\partial}{\partial t}\left(\rho v_{0 r}^{2}\right)+\frac{1}{r^{2}} \frac{\partial\left(r^{2} \rho v_{0 r}^{3}\right)}{\partial r}\right]\right\}\right\} \\
& +\frac{\partial p}{\partial r}-\frac{\partial}{\partial r}\left(\tau \frac{\partial p}{\partial t}\right)-2 \frac{\partial}{\partial r}\left(\tau \frac{1}{r^{2}} \frac{\partial\left(r^{2} p v_{0 r}\right)}{\partial r}\right)-\frac{1}{r^{2}} \frac{\partial}{\partial r}\left(\tau r^{2} \frac{\partial\left(p v_{0 r}\right)}{\partial r}\right)=0
\end{aligned}
$$

Energy equation

$$
\begin{aligned}
& \frac{\partial}{\partial t}\left\{\frac{1}{2} \rho v_{0}^{2}+\frac{3}{2} p-\tau\left[\frac{\partial}{\partial t}\left(\frac{1}{2} \rho v_{0}^{2}+\frac{3}{2} p\right)+\frac{1}{r^{2}} \frac{\partial}{\partial r}\left(r^{2} v_{0 r}\left(\frac{1}{2} \rho v_{0}^{2}+\frac{5}{2} p\right)\right)\right]\right\} \\
& +\frac{1}{r^{2}} \frac{\partial}{\partial r}\left\{r^{2}\left(\frac{1}{2} \rho v_{0}^{2}+\frac{5}{2} p\right) v_{0 r}-\tau\left[\frac{\partial}{\partial t}\left(\left(\frac{1}{2} \rho v_{0}^{2}+\frac{5}{2} p\right) v_{0 r}\right)+\frac{1}{r^{2}} \frac{\partial}{\partial r}\left(r^{2}\left(\frac{1}{2} \rho v_{0}^{2}+\frac{7}{2} p\right) v_{0 r}^{2}\right)\right]\right\}=0
\end{aligned}
$$

For the stationary case we find after some transformations continuity equation

$$
r^{2} \rho v_{0 r}-\tau \frac{\partial\left(r^{2} \rho v_{0 r}^{2}\right)}{\partial r}-\tau r^{2} \frac{\partial p}{\partial r}=0
$$

motion equation

$$
\frac{\partial}{\partial r}\left\{\rho v_{0 r}^{2} r^{2}\right\}-\tau \frac{\partial^{2}}{\partial r^{2}}\left(r^{2} \rho v_{0 r}^{3}\right)+r^{2} \frac{\partial p}{\partial r}-3 \tau r^{2} \frac{\partial^{2}}{\partial r^{2}}\left(p v_{0 r}\right)-6 \tau r \frac{\partial}{\partial r}\left(p v_{0 r}\right)+8 \tau p v_{0 r}=0,
$$

energy equation

$$
r^{2}\left(\rho v_{0}^{2}+5 p\right) v_{0 r}-\tau \frac{\partial}{\partial r}\left(r^{2}\left(\rho v_{0}^{2}+7 p\right) v_{0 r}^{2}\right)=0
$$

Obviously continuity equation (3.6) and energy equation can be integrated once in the stationary case (see (3.10), (3.11)). For the following application of the numerical methods it is reasonable to continue the transformation of Eqs. (3.9) - (3.11). We find:

continuity equation

momentum equation

$$
r \rho v_{0 r}-2 \tau \rho v_{0 r}^{2}-2 \tau r \rho v_{0 r} \frac{\partial v_{0 r}}{\partial r}-\tau r v_{0 r}^{2} \frac{\partial \rho}{\partial r}-\tau r \frac{\partial p}{\partial r}=0
$$

$$
\begin{aligned}
& \tau^{2} r^{2} \frac{\partial^{2}}{\partial r^{2}}\left(\rho v_{0 r}^{3}\right)+4 \tau^{2} r \frac{\partial}{\partial r}\left(\rho v_{0 r}^{3}\right)+2 \tau^{2} \rho v_{0 r}^{3} \\
& +3 \tau^{2} r^{2} \frac{\partial^{2}}{\partial r^{2}}\left(p v_{0 r}\right)+6 \tau^{2} r \frac{\partial}{\partial r}\left(p v_{0 r}\right)-8 \tau^{2} p v_{0 r}-r^{2} \rho v_{0 r}=0,
\end{aligned}
$$

energy equation

$$
r\left(\rho v_{0}^{2}+5 p\right)-\tau r\left(14 p+4 \rho v_{0}^{2}\right) \frac{\partial v_{0 r}}{\partial r}-\tau r v_{0 r}^{3} \frac{\partial \rho}{\partial r}-7 \tau r v_{0 r} \frac{\partial p}{\partial r}-2 \tau\left(\rho v_{0}^{2}+7 p\right) v_{0 r}=0
$$

Equation (3.13) is obtained after adding the momentum equation (3.10) multiplied by the coefficient $\tau$ with the continuity equation (3.9). Let us introduce now the new dependent variables

$$
y=\rho v_{0 r}^{3}
$$




$$
z=p v_{0 r}
$$

We reach the system of equations which was used in the following numerical integrations: continuity equation

$$
r y-2 \tau y v_{0 r}-2 \tau r y \frac{\partial v_{0 r}}{\partial r}-\tau r v_{0 r} \frac{\partial y}{\partial r}+3 \tau r y \frac{\partial v_{0 r}}{\partial r}-\tau r v_{0 r} \frac{\partial z}{\partial r}+\tau r z \frac{\partial v_{0 r}}{\partial r}=0
$$

momentum equation

$$
\tau^{2} r^{2} \frac{\partial^{2} y}{\partial r^{2}}+4 \tau^{2} r \frac{\partial y}{\partial r}+2 \tau^{2} y+3 \tau^{2} r^{2} \frac{\partial^{2} z}{\partial r^{2}}+6 \tau^{2} r \frac{\partial z}{\partial r}-8 \tau^{2} z-r^{2} \frac{y}{v_{0 r}^{2}}=0
$$

energy equation

$$
r(y+5 z)-\tau r(7 z+y) \frac{\partial v_{0 r}}{\partial r}-\tau r v_{0 r} \frac{\partial y}{\partial r}-7 \tau r v_{0 r} \frac{\partial z}{\partial r}-2 \tau v_{0 r}(y+7 z)=0
$$

The system of equations $(3.17)$ - (3.19) contains the dependent variables $v_{0 r}, y, z$. We transform (3.17) - (3.19) to the dimensionless form using the scales of length $L$, velocity $V$, time $\tau$, pressure $P$, mass density $R$. The dimensionless equations (3.17) - (3.19) have the form continuity equation

$$
\hat{r} \hat{z} \frac{\partial \hat{v}_{0 r}}{\partial \hat{r}}-2 \hat{r} \hat{y} \frac{\partial \hat{v}_{0 r}}{\partial \hat{r}}-\hat{r} \hat{v}_{0 r} \frac{\partial \hat{y}}{\partial \hat{r}}+3 \hat{r} \hat{y} \frac{\partial \hat{v}_{0 r}}{\partial \hat{r}}-\hat{r} \hat{v}_{0 r} \frac{\partial \hat{z}}{\partial \hat{r}}+\hat{r} \hat{y}-2 \hat{y} \hat{v}_{0 r}=0
$$

momentum equation

$$
\hat{r}^{2} \frac{\partial^{2} \hat{y}}{\partial \hat{r}^{2}}+3 \hat{r}^{2} \frac{\partial^{2} \hat{z}}{\partial \hat{r}^{2}}+4 \hat{r} \frac{\partial \hat{y}}{\partial \hat{r}}+6 \hat{r} \frac{\partial \hat{z}}{\partial \hat{r}}+2 \hat{y}-8 \hat{z}-\hat{r}^{2} \frac{\hat{y}}{\hat{v}_{0 r}^{2}}=0
$$

energy equation

$$
\begin{gathered}
\hat{r}(7 \hat{z}+\hat{y}) \frac{\partial \hat{v}_{0 r}}{\partial \hat{r}}+\hat{r} \hat{v}_{0 r} \frac{\partial \hat{y}}{\partial \hat{r}}+7 \hat{r} \hat{v}_{0 r} \frac{\partial \hat{z}}{\partial \hat{r}}+2 \hat{v}_{0 r}(\hat{y}+7 \hat{z})-\hat{r}(\hat{y}+5 \hat{z})=0 \\
\hat{y}=\hat{\rho} \hat{v}_{0 r}^{3} \\
\hat{z}=\hat{p} \hat{v}_{0 r}
\end{gathered}
$$

\section{About Stability of Levitating Objects}

We will use the following statement of the problem. The spherical levitating object is placed in the space. A perturbation takes place on the surface $(\hat{r}=1)$ of this object. What can be said about stability of this object? This statement belongs to the class of Cauchy problems. If $\hat{v}_{0 r}(\hat{r})=0$, then the object is stable and Eqs. (3.20) - (3.22) are satisfied identically. Let us introduce the radial perturbations on the surface of the object. Now we are ready to display the results of the mathematical modeling realized with the help of Maple (the versions Maple 9 or more can be used). The system (3.20) - (3.22) has the great possibilities of mathematical modeling as the result of changing the six Cauchy conditions describing the character features of physical system.

Maple program contains Maple's notations - for example the expression $D\left(\tilde{v}_{0 r}\right)(1)=1$ means in the usual notations $\left(\partial \tilde{v}_{0 r} / \partial \tilde{r}\right)(1)=1$, independent variable $t$ responds to $\tilde{r}$. The following Maple notations on figures are used: v- velocity $\hat{v}_{0 r}, \mathrm{p}$ - self-consistent pressure $\hat{p}$, and $\mathrm{r}$ - the density $\hat{\rho}, \mathrm{T}$ $\tilde{\tau}=1$. Explanations placed under all following figures. The results of the calculations are presented in figures 4.1 - 4.16. We use the following lines: $\tilde{v}_{0 r}$ - solid line, $\hat{p}$ - dashed line, $\hat{\rho}$ - dotted line. The information required is contained in the figures and in figure captions. The Cauchy conditions are written under each figure, these conditions can be changed widely. The upper label contains information about the limits of the solution existence. 
Figures $4.4,4.8,4.12,4.16$ contain the space evolution of the ratio $\hat{p} / \hat{\rho}$, which is proportional to the temperature near the object surface. The left and right boundaries of the solution existence are indicated as $\lim 1$ and lim2 correspondingly.

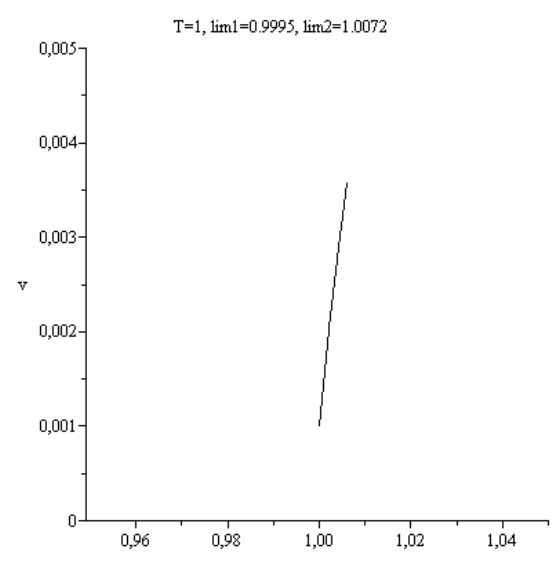

Figure. 4.1. Evolution of $\hat{v}_{0 r}(\tilde{r})$;

$\hat{v}_{0 r}(1)=10^{-3}, \hat{y}(1)=10^{-9}, \hat{z}(1)=10^{-3}, D\left(\hat{v}_{0 r}\right)(1)=1, D(\hat{y})(1)=1, D(\hat{z})(1)=1, \quad \hat{p}(1)=1, \quad \hat{\rho}(1)=1$

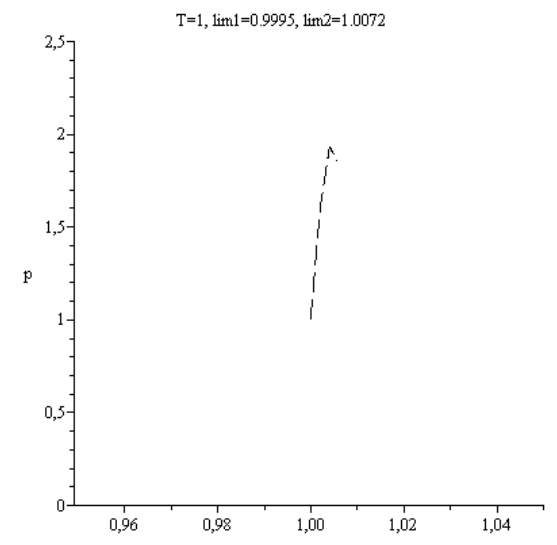

Figure 4.2. Evolution of $\hat{p}(\tilde{r})$;

$\hat{v}_{0 r}(1)=10^{-3}, \hat{y}(1)=10^{-9}, \hat{z}(1)=10^{-3}, \quad D\left(\hat{v}_{0 r}\right)(1)=1, \quad D(\hat{y})(1)=1, D(\hat{z})(1)=1, \quad \hat{p}(1)=1, \quad \hat{\rho}(1)=1$.

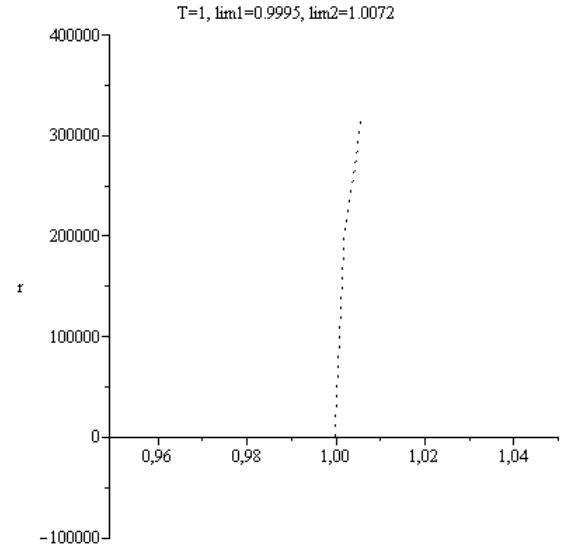

Figure 4.3. Evolution of $\hat{\rho}(\tilde{r})$;

$\hat{v}_{0 r}(1)=10^{-3}, \hat{y}(1)=10^{-9}, \hat{z}(1)=10^{-3}, D\left(\hat{v}_{0 r}\right)(1)=1, D(\hat{y})(1)=1, D(\hat{z})(1)=1, \hat{p}(1)=1, \hat{\rho}(1)=1$. 


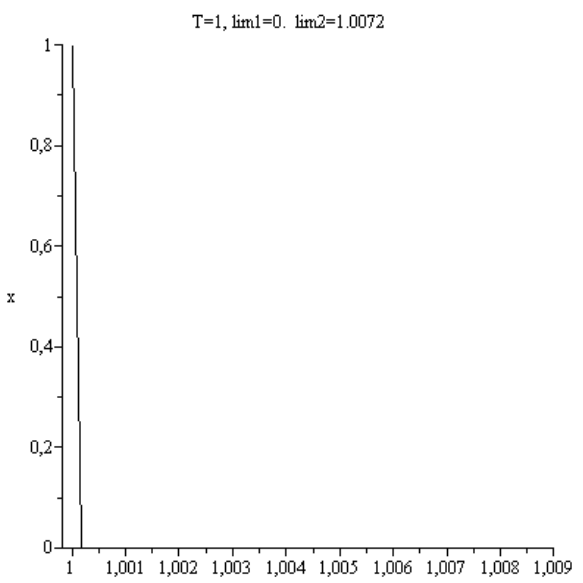

Figure 4.4. Evolution of $\hat{p}(\hat{r}) / \hat{\rho}(\tilde{r})$;

$\hat{v}_{0 r}(1)=10^{-3}, \hat{y}(1)=10^{-9}, \hat{z}(1)=10^{-3}, D\left(\hat{v}_{0 r}\right)(1)=1, D(\hat{y})(1)=1, D(\hat{z})(1)=1, \hat{p}(1)=1, \quad \hat{\rho}(1)=1$.

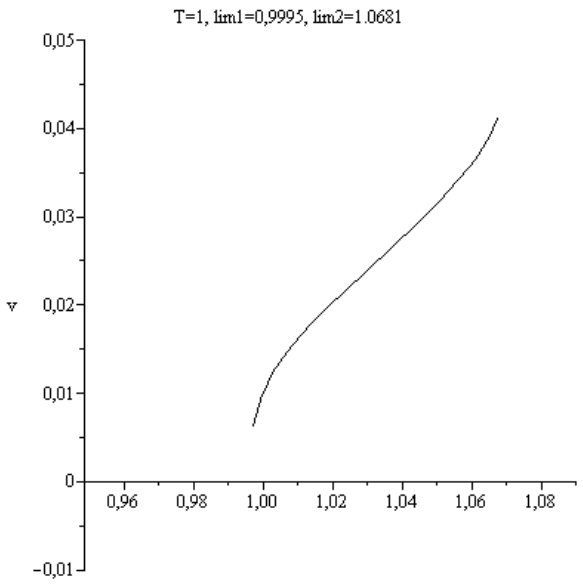

Figure 4.5. Evolution of $\hat{v}_{0 r}(\tilde{r})$;

$\hat{v}_{0 r}(1)=10^{-2}, \hat{y}(1)=10^{-6}, \hat{z}(1)=10^{-2}, D\left(\hat{v}_{0 r}\right)(1)=1, D(\hat{y})(1)=1, D(\hat{z})(1)=1, \hat{p}(1)=1, \quad \hat{\rho}(1)=1$.

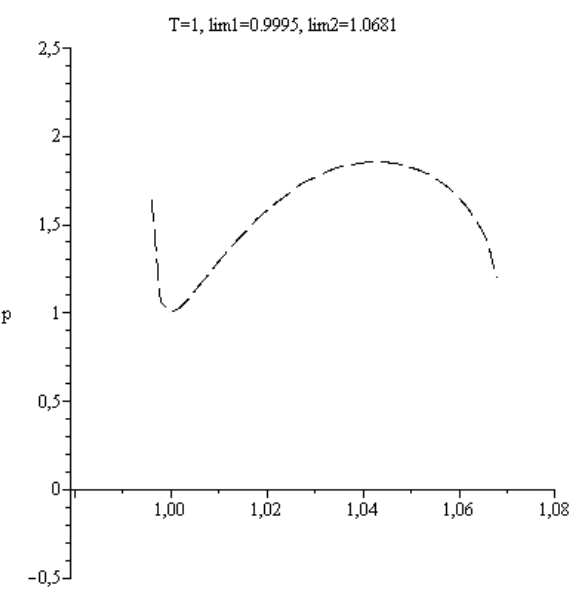

Figure 4.6. Evolution of $\hat{p}_{0 r}(\tilde{r})$;

$\hat{v}_{0 r}(1)=10^{-2}, \hat{y}(1)=10^{-6}, \hat{z}(1)=10^{-2}, D\left(\hat{v}_{0 r}\right)(1)=1, D(\hat{y})(1)=1, D(\hat{z})(1)=1, \hat{p}(1)=1, \quad \hat{\rho}(1)=1$. 


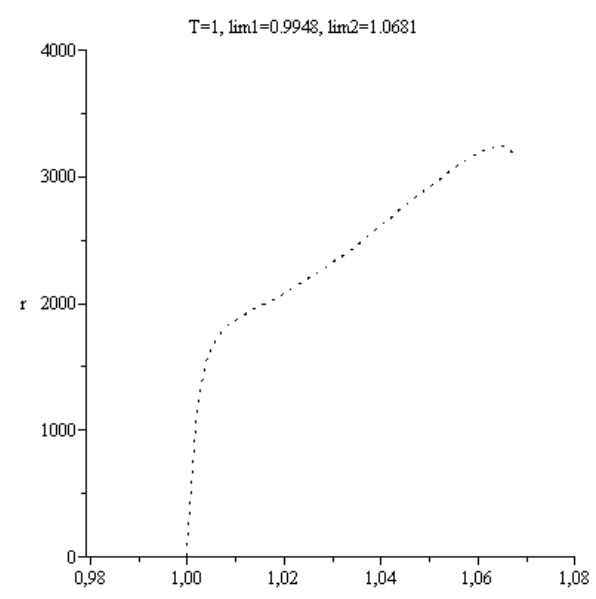

Figure 4.7. Evolution of $\hat{\rho}(\tilde{r})$;

$\hat{v}_{0 r}(1)=10^{-2}, \hat{y}(1)=10^{-6}, \hat{z}(1)=10^{-2}, D\left(\hat{v}_{0 r}\right)(1)=1, D(\hat{y})(1)=1, D(\hat{z})(1)=1, \hat{p}(1)=1, \quad \hat{\rho}(1)=1$.

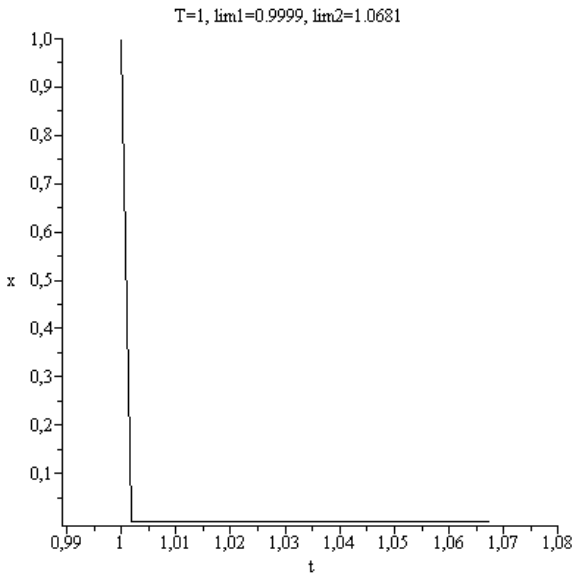

Figure 4.8. Evolution of $\hat{p}(\hat{r}) / \hat{\rho}(\tilde{r})$;

$\hat{v}_{0 r}(1)=10^{-2}, \hat{y}(1)=10^{-6}, \hat{z}(1)=10^{-2}, D\left(\hat{v}_{0 r}\right)(1)=1, D(\hat{y})(1)=1, D(\hat{z})(1)=1, \quad \hat{p}(1)=1, \quad \hat{\rho}(1)=1$.

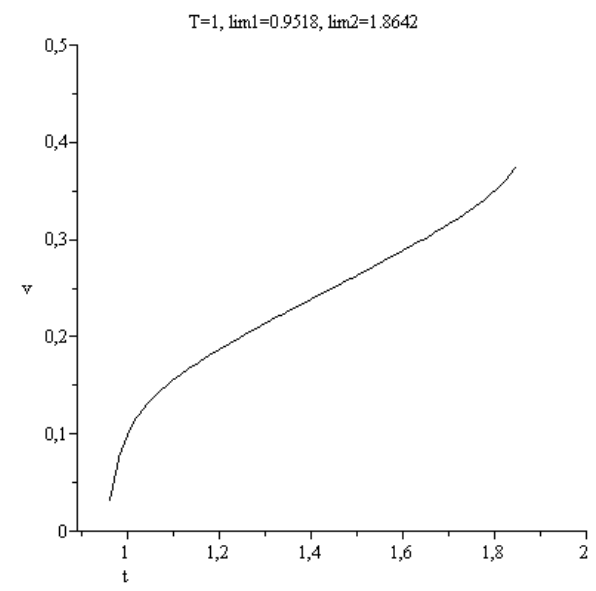

Figure 4.9. Evolution of $\hat{v}_{0 r}(\tilde{r})$;

$\hat{v}_{0 r}(1)=10^{-1}, \hat{y}(1)=10^{-3}, \hat{z}(1)=10^{-1}, D\left(\hat{v}_{0 r}\right)(1)=1, D(\hat{y})(1)=1, D(\hat{z})(1)=1, \hat{p}(1)=1, \hat{\rho}(1)=1$. 


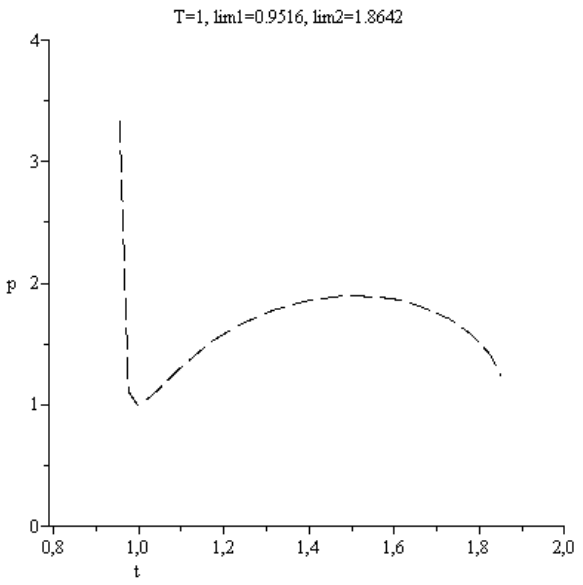

Figure 4.10. Evolution of $\hat{p}(\tilde{r})$;

$\hat{v}_{0 r}(1)=10^{-1}, \hat{y}(1)=10^{-3}, \hat{z}(1)=10^{-1}, D\left(\hat{v}_{0 r}\right)(1)=1, D(\hat{y})(1)=1, D(\hat{z})(1)=1, \hat{p}(1)=1, \hat{\rho}(1)=1$.

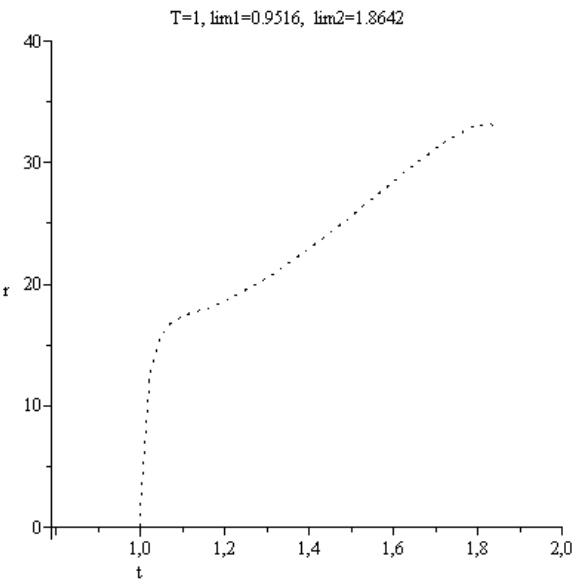

Figure 4.11. Evolution of $\hat{\rho}(\tilde{r})$

$\hat{v}_{0 r}(1)=10^{-1}, \hat{y}(1)=10^{-3}, \hat{z}(1)=10^{-1}, D\left(\hat{v}_{0 r}\right)(1)=1, D(\hat{y})(1)=1, D(\hat{z})(1)=1, \hat{p}(1)=1, \hat{\rho}(1)=1$.

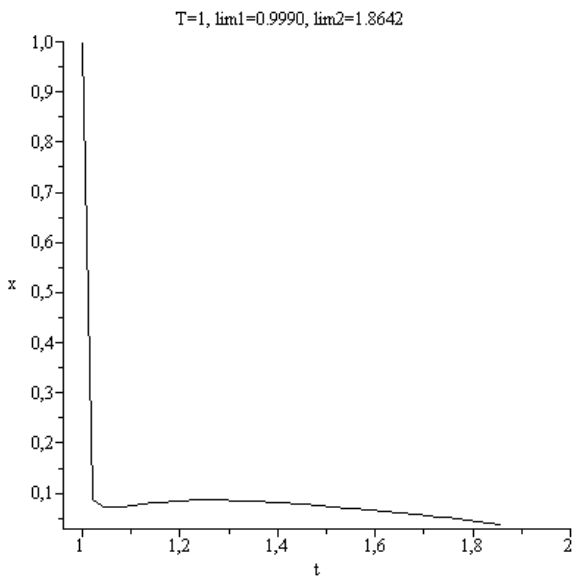

Figure 4.12. Evolution of $\hat{p}(\hat{r}) / \hat{\rho}(\tilde{r})$;

$\hat{v}_{0 r}(1)=10^{-1}, \hat{y}(1)=10^{-3}, \hat{z}(1)=10^{-1}, D\left(\hat{v}_{0 r}\right)(1)=1, D(\hat{y})(1)=1, D(\hat{z})(1)=1, \hat{p}(1)=1, \hat{\rho}(1)=1$. 


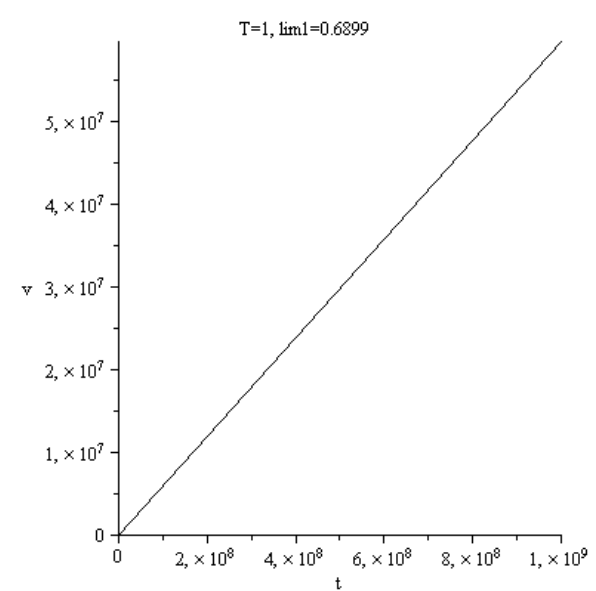

Figure 4.13. Evolution of $\hat{v}_{0 r}(\tilde{r})$; $\hat{v}_{0 r}(1)=1, \hat{y}(1)=1, \hat{z}(1)=1, D\left(\hat{v}_{0 r}\right)(1)=1, D(\hat{y})(1)=1, D(\hat{z})(1)=1, \hat{p}(1)=1, \hat{\rho}(1)=1$.

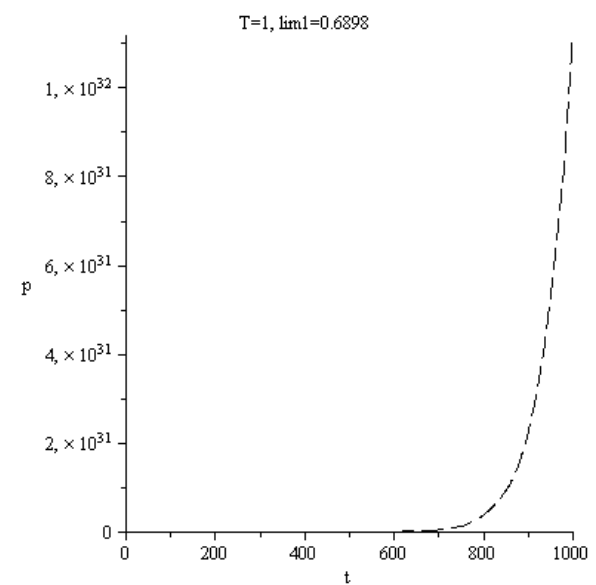

Figure 4.14. Evolution of $\hat{p}(\tilde{r})$;

$\hat{v}_{0 r}(1)=1, \hat{y}(1)=1, \hat{z}(1)=1, D\left(\hat{v}_{0 r}\right)(1)=1, D(\hat{y})(1)=1, \quad D(\hat{z})(1)=1, \quad \hat{p}(1)=1, \quad \hat{\rho}(1)=1$.

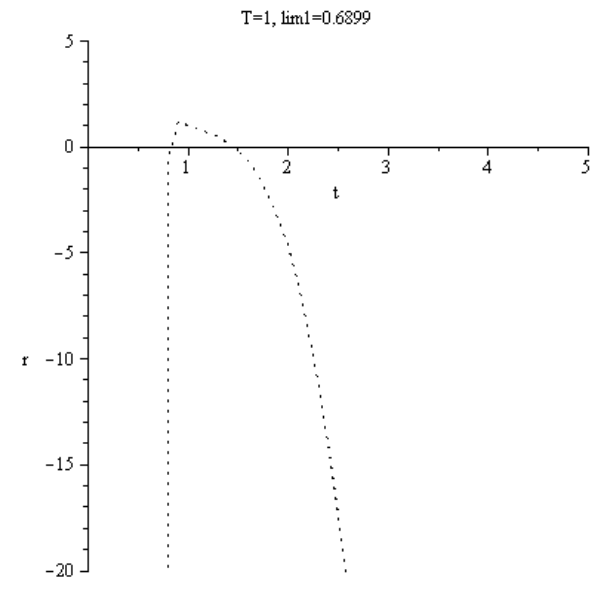

Figure 4.15. Evolution of $\hat{\rho}(\tilde{r})$;

$\hat{v}_{0 r}(1)=1, \hat{y}(1)=1, \hat{z}(1)=1, D\left(\hat{v}_{0 r}\right)(1)=1, D(\hat{y})(1)=1, D(\hat{z})(1)=1, \hat{p}(1)=1, \quad \hat{\rho}(1)=1$. 


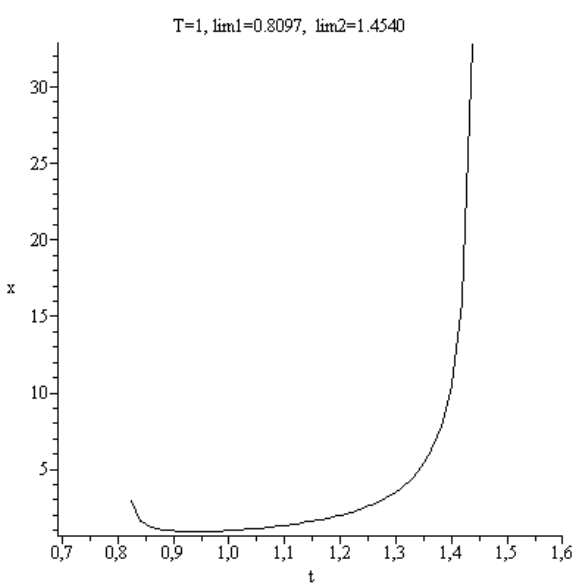

Figure 4.16. Evolution of $\hat{p}(\hat{r}) / \hat{\rho}(\tilde{r})$;

$\hat{v}_{0 r}(1)=1, \hat{y}(1)=1, \hat{z}(1)=1, D\left(\hat{v}_{0 r}\right)(1)=1, D(\hat{y})(1)=1, D(\hat{z})(1)=1, \hat{p}(1)=1, \hat{\rho}(1)=1$.

\section{Necessary Conditions for the Stability}

Let us consider now in details the second part of the problem which was formulated at the beginning of section 3. Namely we transform the general stability conditions (2.7), (2.13) and (2.15) for the simplest stationary case when $\mathbf{v}_{0}=$ const. In other words we obtain the necessary conditions defining the configuration of the electro-magnetic field for the stable object. Then if $\mathbf{v}_{0}=0$ one obtains from (2.7), (2.13) and (2.15) correspondingly

$$
\begin{gathered}
\sum_{\alpha} \tau_{\alpha}^{(0)}\left[\rho_{\alpha} \mathbf{F}_{\alpha}^{(1)}+\frac{q_{\alpha}}{m_{\alpha}} \rho_{\alpha} \mathbf{v}_{0} \times \mathbf{B}\right]=\mathbf{L}(t), \\
\tau_{\alpha}^{(0)}\left[\rho_{\alpha} \mathbf{F}_{\alpha}^{(1)}+\frac{q_{\alpha}}{m_{\alpha}} \rho_{\alpha} \mathbf{v}_{0} \times \mathbf{B}\right]=\mathbf{L}_{\alpha}(t), \\
\sum_{\alpha} \mathbf{F}_{\alpha}^{(1)} \rho_{\alpha}^{a}=\frac{\partial}{\partial t} \mathbf{L}(t)-\sum_{\alpha} \frac{q_{\alpha}}{m_{\alpha}} \mathbf{L}_{\alpha}(t) \times \mathbf{B}+\mathbf{v}_{0}\left(\frac{\partial}{\partial \mathbf{r}} \cdot \mathbf{L}(t)\right)+\left(\mathbf{v}_{0} \cdot \frac{\partial}{\partial \mathbf{r}}\right) \mathbf{L}(t) . \\
\mathbf{v}_{0} \cdot \sum_{\alpha} \mathbf{F}_{\alpha}^{(1)} \rho_{\alpha}^{a}=\frac{\partial}{\partial t} \sum_{\alpha} \tau_{\alpha}^{(0)}\left(\rho_{\alpha} \mathbf{F}_{\alpha}^{(1)} \cdot \mathbf{v}_{0}\right)+\sum_{\alpha} \tau_{\alpha}^{(0)} \mathbf{F}_{\alpha}^{(1)} \cdot\left[\frac{\partial}{\partial \mathbf{r}} \cdot p_{\alpha} \overrightarrow{\mathrm{I}}\right] \\
+\frac{\partial}{\partial \mathbf{r}} \cdot\left\{\sum_{\alpha} \tau_{\alpha}^{(0)}\left[\begin{array}{c}
\left.\rho_{\alpha} \mathbf{F}_{\alpha}^{(1)} \cdot \mathbf{v}_{0} \mathbf{v}_{0}+p_{\alpha} \mathbf{F}_{\alpha}^{(1)} \cdot \overrightarrow{\mathrm{I}}+\frac{1}{2} \rho_{\alpha} v_{0}^{2} \mathbf{F}_{\alpha}^{(1)}+\frac{3}{2} \mathbf{F}_{\alpha}^{(1)} p_{\alpha}+\frac{\rho_{\alpha} v_{0}^{2}}{2} \frac{q_{\alpha}}{m_{\alpha}}\left[\mathbf{v}_{0} \times \mathbf{B}\right]\right] \\
+\frac{5}{2} p_{\alpha} \frac{q_{\alpha}}{m_{\alpha}}\left[\mathbf{v}_{0} \times \mathbf{B}\right] \\
+\frac{\partial}{\partial \mathbf{r}} \cdot \sum_{\alpha} \varepsilon_{\alpha} n_{\alpha} \frac{1}{\rho_{\alpha}} \mathbf{L}_{\alpha}-\sum_{\alpha} \frac{1}{\rho_{\alpha}} \mathbf{F}_{\alpha}^{(1)} \cdot \mathbf{L}_{\alpha}
\end{array}\right]\right\}
\end{gathered}
$$

If in the first approximation $\mathbf{v}_{0}=0$ and $\tau_{\alpha}^{(0)}=\tau=$ const, we have from (5.1) - (5.4) for the stationary case

$$
\begin{gathered}
\mathbf{L}_{\alpha}=\tau \rho_{\alpha} \mathbf{F}_{\alpha}^{(1)} \\
\sum_{\alpha} \mathbf{F}_{\alpha}^{(1)} \rho_{\alpha}^{a}=-\tau \sum_{\alpha} q_{\alpha} n_{\alpha}\left[\mathbf{F}_{\alpha}^{(1)} \times \mathbf{B}\right] \\
\sum_{\alpha} \mathbf{F}_{\alpha}^{(1)} \cdot\left[\frac{\partial}{\partial \mathbf{r}} \cdot p_{\alpha} \overrightarrow{\mathrm{I}}\right]+\frac{\partial}{\partial \mathbf{r}} \cdot \sum_{\alpha}\left[\mathbf{F}_{\alpha}^{(1)}\left(\frac{5}{2} p_{\alpha}+\varepsilon_{\alpha} n_{\alpha}\right)\right]=\sum_{\alpha} \mathbf{F}_{\alpha}^{(1)} \cdot \mathbf{F}_{\alpha}^{(1)}
\end{gathered}
$$


where $\mathbf{F}_{\alpha}^{(1)}$ is a force of the non-magnetic origin acting on the particle $\alpha$-species in the unit of volume, $\varepsilon_{\alpha} n_{\alpha}$ - the internal energy of the particles $\alpha$-species in the volume unit. If the force $\mathbf{F}_{\alpha}^{(1)}$ is connected only with the electric field and gravitation we find

$$
\mathbf{F}_{\alpha}^{(1)}=\rho_{\alpha} \mathbf{g}+q_{\alpha} n_{\alpha} \mathbf{E}
$$

If we intend to take into account only the electron component, then

$$
\begin{gathered}
\mathbf{F}=\tau \frac{e}{m_{e}}[\mathbf{F} \times \mathbf{B}] \\
\mathbf{F} \cdot\left[\frac{\partial}{\partial \mathbf{r}} \cdot p_{e} \overrightarrow{\mathrm{I}}\right]+\frac{5}{2} \frac{\partial}{\partial \mathbf{r}} \cdot \mathbf{F} p_{e}=\mathbf{F} \cdot \mathbf{F} \\
\mathbf{F}=\rho_{e} \mathbf{g}-e n_{e} \mathbf{E}
\end{gathered}
$$

where $e=1.602176 \cdot 10^{-19} \mathrm{C}$ is the absolute value of the electron charge, $\frac{e}{m_{e}}=1.758819 \cdot 10^{11} \frac{C}{\mathrm{~kg}}$, $m_{e}=9,109382 \cdot 10^{-31} \mathrm{~kg}$.

In the spherical coordinate system (5.10) is written as

$$
\frac{5}{r} p_{e} F_{r}+\frac{5}{2} p_{e} \frac{\partial F_{r}}{\partial r}+\frac{7}{2} F_{r} \frac{\partial p_{e}}{\partial r}=F_{r}^{2}
$$

Important remarks:

1. In general case we need to solve the system of equations (2.1) - (2.3) without forces (see also (3.1) (3.8) and (2.7), (2.13) and (2.15) defining the system of the sufficient conditions for levitation. This combination of equations defines not only the hydrodynamic parameters but also the self consistent forces.

2. In the simplest case $\mathbf{v}_{0}=0$ and $\tau_{\alpha}^{(0)}=\tau=$ const equation (5.9) leads to the trivial solution $\mathbf{F}=0$ and obvious condition of levitation $\rho_{e} \mathbf{g}=e n_{e} \mathbf{E}$.

3. Much more interesting situation appears when the magnetic field involves into consideration with condition $\mathbf{v}_{0} \neq 0$. This case returns us to the Item 1 in Section 3. But for the simplification of the numerical calculations the iteration process can be realized with the preliminary $F_{r}$ calculation and following definition of velocity using (5.3).

Following this scheme let us add Eq. (5.12) to the system of equations (3.20) - (3.24) with the aim to reveal the character features of the force evolution. The results of calculations are reflected on figures (5.1) - (5.6).

Figures 5.1 - 5.6 show the typical "walls" obtained as a result of the self-consistent solution.

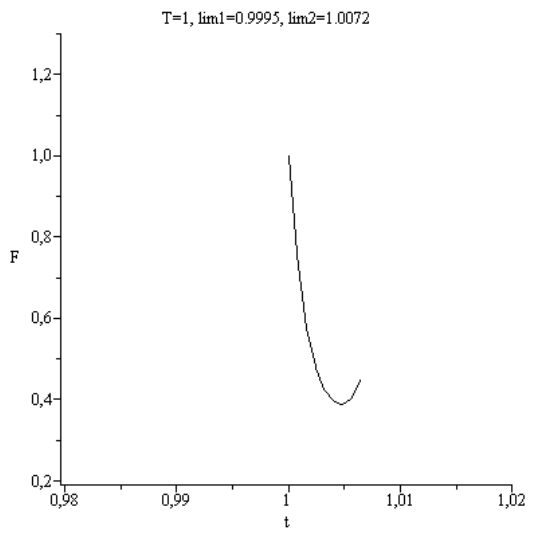

Figure 5.1. Evolution of $\hat{F}_{r}(\hat{r})$;

$\hat{v}_{0 r}(1)=10^{-3}, \hat{y}(1)=10^{-9}, \hat{z}(1)=10^{-3}, D\left(\hat{v}_{0 r}\right)(1)=1, D(\hat{y})(1)=1, D(\hat{z})(1)=1, \hat{p}(1)=1, \quad \hat{\rho}(1)=1, \hat{F}_{r}(1)=1$ 


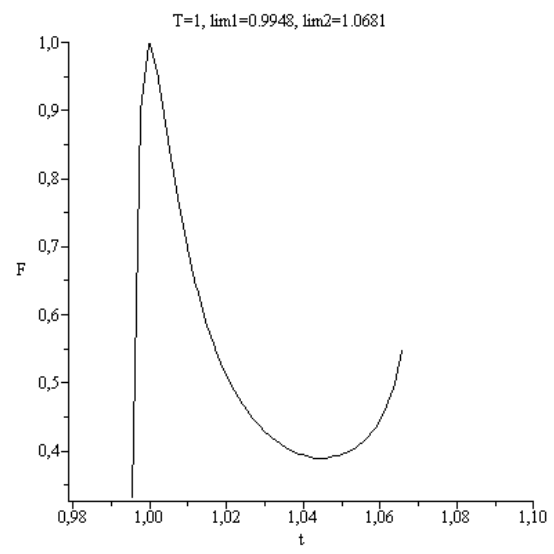

Figure 5.2. Evolution of $\hat{F}_{r}(\hat{r})$;

$\hat{v}_{0 r}(1)=10^{-2}, \hat{y}(1)=10^{-6}, \hat{z}(1)=10^{-2}, D\left(\hat{v}_{0 r}\right)(1)=1, D(\hat{y})(1)=1, D(\hat{z})(1)=1, \hat{p}(1)=1, \quad \hat{\rho}(1)=1, \hat{F}_{r}(1)=1$

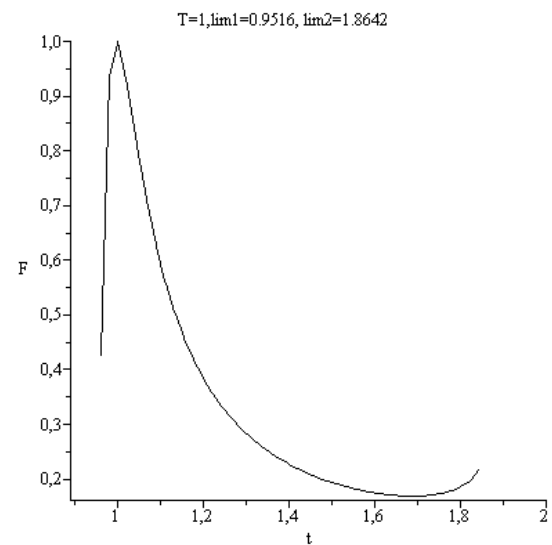

Figure 5.3. Evolution of $\hat{F}_{r}(\hat{r})$;

$\hat{v}_{0 r}(1)=0.1, \hat{y}(1)=10^{-3}, \hat{z}(1)=0.1, D\left(\hat{v}_{0 r}\right)(1)=1, D(\hat{y})(1)=1, D(\hat{z})(1)=1, \hat{p}(1)=1, \quad \hat{\rho}(1)=1, \hat{F}_{r}(1)=1$ $T=1$, lim1 $=0.8725$

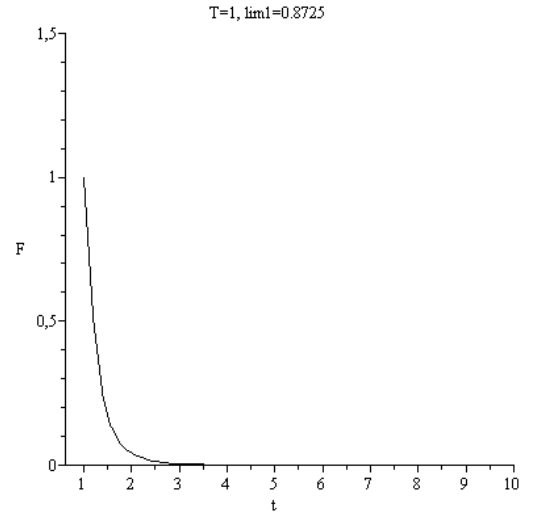

Figure 5.4. Evolution of $\hat{F}_{r}(\hat{r})$;

$\hat{v}_{0 r}(1)=0.3, \hat{y}(1)=27 \cdot 10^{-3}, \hat{z}(1)=0.3, D\left(\hat{v}_{0 r}\right)(1)=1, D(\hat{y})(1)=1, D(\hat{z})(1)=1, \hat{p}(1)=1, \hat{\rho}(1)=1, \hat{F}_{r}(1)=1$ 


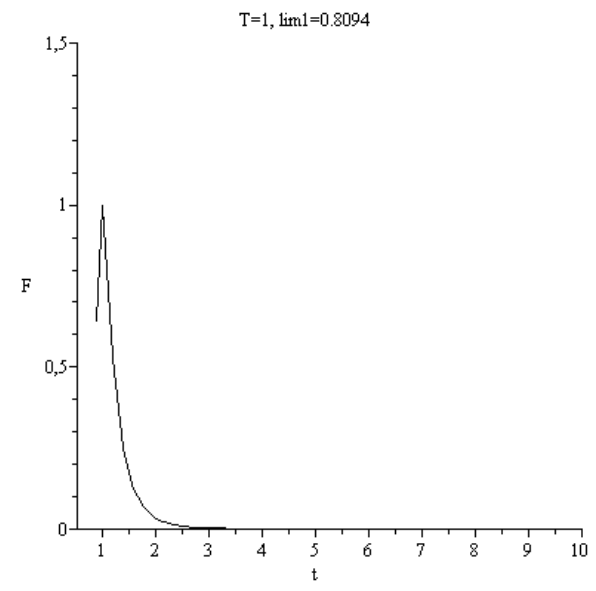

Figure 5.5. Evolution of $\hat{F}_{r}(\hat{r})$;

$\hat{v}_{0 r}(1)=\frac{1}{2}, \hat{y}(1)=\frac{1}{8}, \hat{z}(1)=\frac{1}{2}, D\left(\hat{v}_{0 r}\right)(1)=1, D(\hat{y})(1)=1, D(\hat{z})(1)=1, \hat{p}(1)=1, \quad \hat{\rho}(1)=1, \hat{F}_{r}(1)=1$

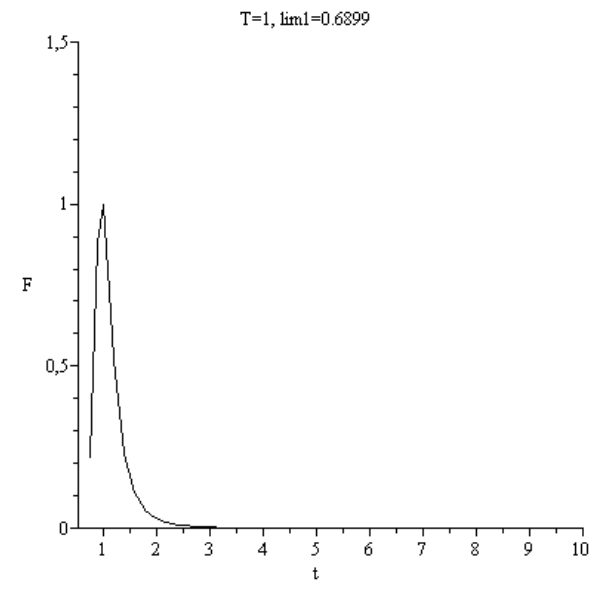

Figure 5.6. Evolution of $\hat{F}_{r}(\hat{r})$;

$\hat{v}_{0 r}(1)=1, \hat{y}(1)=1, \hat{z}(1)=1, D\left(\hat{v}_{0 r}\right)(1)=1, D(\hat{y})(1)=1, \quad D(\hat{z})(1)=1, \quad \hat{p}(1)=1, \quad \hat{\rho}(1)=1, \hat{F}_{r}(1)=1$,

The discussed stability conditions should be fulfilled for all types of convertors including [16].

It is known from Internet so called flying "Searl discs". Descriptions of these flights sound fantastic. Less impressive but well-documented experiments were performed by V.V. Roschin and S.M. Godin [16], (see Figure 6.6.8). The first version of the Converter created by Roschin and Godin is made on the same principle as the Searle generator. Elements of the Converter construction [16] contain stator magnets, stator, rotating magnetic rollers, grid electrode and high voltage source; in [16] it is equal to $20 \mathrm{kV}$. Rollers rotate on a stator and there is a force directed on an axis of rotation. 


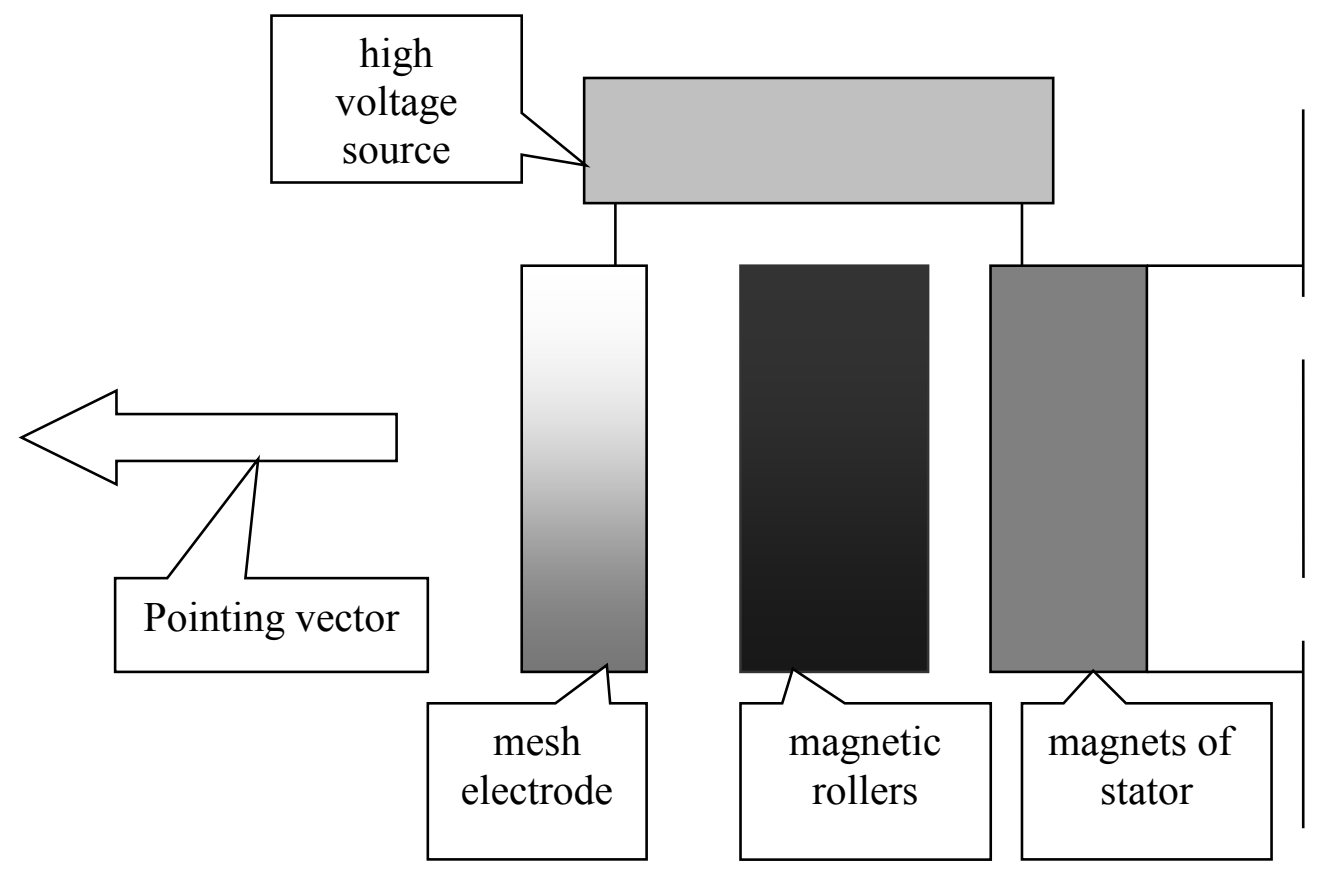

Figure 5.7. Elements of the Converter construction.

They write [16]: "The installation was started by the promotion of the rotor with the help of an electric motor. Revolutions gradually increased as long as the ammeter included in the motor power circuit, did not start to show a zero value of the current consumed and the presence of reverse current. This corresponded to about $550 \mathrm{rpm}$, and the magnetic displacement sensor of the platform will begin to record the change in weight of the platform is already at 200rpm. ...

At a maximum output power of $7 \mathrm{~kW}$, the weight change of the entire platform weighing $350 \mathrm{~kg}$ reaches $35 \%$ of the weight in a stationary state" $\ldots$

The effect of changing the weight is reversible relative to the direction of rotation of the rotor, and has some hysteresis. When rotating clockwise, the critical mode occurs in the area of 550rpm and the thrust is created against the direction of the gravity vector, and when rotating anticlockwise, the critical mode occurs in the area of 600rpm and the thrust is created in the direction of the gravity vector."

Let us explain these phenomena from the position of nonlocal physics. The calculations reflected on figures 5.1 - 5.6 with the "wall appearance" allow considering the relation (5.10) as follows:

$$
\mathbf{F}_{d e c}\left(\mathbf{r}, \mathbf{v}_{0}\right)=\tau e n_{e}\left\{[\mathbf{g} \times \mathbf{B}]-\frac{e}{m_{e}}[\mathbf{E} \times \mathbf{B}]\right\}
$$

Relation (5.13) leads to the appearance two energy flux densities - Pointing flux and Alexeev flux denoted here correspondingly as

$$
\mathbf{S}_{P}=[\mathbf{E} \times \mathbf{B}]
$$

and

$$
\mathbf{S}_{A}=[\mathbf{g} \times \mathbf{B}]
$$

As a result the relation (6.6.13) can be written as

$$
\mathbf{F}_{d e c}\left(\mathbf{r}, \mathbf{v}_{0}\right)=\tau e n_{e}\left\{\mathbf{S}_{A}-\frac{e}{m_{e}} \mathbf{S}_{P}\right\}
$$


The sign in front of component of the magnetic induction $\mathbf{B}$ in the vector product $\mathbf{S}_{P}$ depends on the direction of rotation. Relation (5.16) has the transparent physical sense. As a result the forces of the electro-magnetic field delete the influence of gravitation.

Decompensate flux $\mathbf{F}_{d e c}\left(\mathbf{r}, \mathbf{v}_{0}\right)$ defines radial "magnetic walls" using the terminology [16]. The following conclusions of the principal significance can be done:

1. The levitating object remains in the stable state after the small radial perturbations, (figures 4.1 4.12).

2. The small radial perturbations lead to appearance of the layer of the finite width in the vicinity of sphere. This layer has a character feature - the smaller the disturbance, the greater the density of the layer (figures 4.3, 4.7, and 4.11). In the case of small perturbations this very thin layer has the extremely high density (see figure 4.3 ).

3. The small perturbations lead to appearance of the cold layer near the sphere (see figures 4.4, 4.8, 4.12, 4.16). The perturbations lead also to "the walls" of the electro-magnetic origin. These effects were observed in the real experiments [16].

4. Strong perturbations lead to the loss of stability and to the explosion of the object (figures $4.13-4.16$ ) including the Hubble effect (figure 4.13).

5. The effect of the negative collective density takes place for the strong disturbances. This effect is known in the experimental physics [17 - 20]. For the levitation problem this effect needs in the additional investigation.

6. The necessary conditions of the levitation stability (see also Eqs. (3.1) - (3.8) and (2.7), (2.13), (2.15)) lead to the influence of gravitation phenomena on electromagnetism and vice versa. The strict theory of levitation can be constructed only in the frame of non-local physics.

\section{References}

1. Alexeev, B.V. The Generalized Boltzmann Equation, Generalized Hydrodynamic Equations and their Applications. Phil. Trans. Roy. Soc. Lond. 349 p.417-443 (1994)

2. Alexeev, B.V. Generalized Boltzmann physical kinetics. - Amsterdam: Elsevier, 2004. 376 p.

3. Alexeev, B.V. Generalized quantum hydrodynamics and principles of non-local physics. J. of Nanoelectronics and Optoelectronics. (2008) No. 3. P. 143-158.

4. Alexeev, B.V. Application of Generalized Quantum Hydrodynamics in the Theory of Quantum Soliton Evolution, J. Nanoelectron. Optoelectron. (2008) No. 3, p. 316 -328.

5. Earnshaw, S, On the nature of the molecular forces which regulate the constitution of the luminiferous ether, Trans. Cambridge Phil. Soc. 7, 97-112, 1842.

6. Berry, M.V. and Geim, A.K., "Of flying frogs and levitrons", Euro. J. Phys., 18, 307-313 (1997) and http://wwwhfml. sci.kun.nl/hfml/levitate.html.

7. Geim, A.K., "Everyone's Magnetism", Phys. Today, 51 Sept., 36-39 (1998).

8. Geim, A. K, M. Simon, M.D., Boamfa, M.I. and Heflinger, O.L., "Magnet levitation at your fingertips", Nature, 400, 323-324 (1999).

9. Simon, M.D, Geim, A.K. "Diamagnetic levitation; Flying frogs and floating magnets", J. App. Phys., 87, 6200$6204(2000)$.

10. Berry, M.V. The LEVITRON and adiabatic trap for spins, Proc. Roy Soc. Lond., A (1996) 452, 1207-1220.

11. Harrigan, R.M., Levitation device, U.S. Patent \$,382245, May 3, 1983.

12. Alexeev B.V., Unified Non-local Theory of Transport Processes, Elsevier Amsterdam, The Netherlands (2015) $644 \mathrm{p}$.

13. Alexeev B.V., Unified Non-local Relativistic Theory of Transport Processes, Elsevier Amsterdam, The Netherlands (2016) 455p.

14. Alexeev B.V., Nonlocal Astrophysics. Dark matter, Dark Energy, Physical Vacuum. Elsevier Amsterdam, the Netherlands (2017) 454p.

15. Alexeev B.V., To the nonlocal theory of levitation. International Journal of Astronomy, Astrophysics and Space Science 2014; 1(5): 52-58. Published online January 20, 2015 (http://www.openscienceonline.com/journal/aass) 
16. Рощин В.В., Годин С.М., Экспериментальное исследование физических эффектов в динамической магнитной системе. (Experimental investigation of physical effects in the dynamical magnetic system) Письма в ЖтФ, 2000, том 26, вып. 24, стр. 70-75.

17. Forward R.L., Negative Matter Propulsion, Journal of Propulsion and Power, Vol. 6, No., 1, 1990, pp. 28-37

18. Forward R.L., Propellantless Propulsion with Negative Matter Generated by Electric Charges, Joint Pripulsion Conference, July 14-17, 2013, San Jose, CA, 49 $9^{\text {th }}$ AIAA/ASME/SAE/ASEE Joint Propulsion Conference, AIAA 2013-3913, pp. 1-12.

19. Junginger J.E., and Popovic, Z.D., An experimental investigation of the influence of an electrostatic potential on electron mass as predicted by Weber's force law, Can. J. Phys., Vol. 82, 2004, pp. 731-735

20. Assis A.K.T., Changing the Inertial Mass of a Charged Particle, Journal of the Physical Society of Japan, Vol. 62, No. 5, 1993, pp. 1418-1422. 